\title{
Molecular Science of Lubricant Additives ${ }^{\dagger}$
}

\author{
Ichiro Minami \\ Division of Machine Elements, Luleå University of Technology, 97187 Luleå, Sweden; ichiro.minami@ltu.se \\ † Dedicated to the late Professor Keiji Yamamoto in memory of his contributions to organo-silicon chemistry. \\ Academic Editor: Jun Kubota \\ Received: 8 March 2017; Accepted: 21 April 2017; Published: 28 April 2017
}

\begin{abstract}
This review aims at introducing an engineering field of lubrication to researchers who are not familiar with tribology, thereby emphasizing the importance of lubricant chemistry in applied science. It provides initial guidance regarding additive chemistry in lubrication systems for researchers with different backgrounds. The readers will be introduced to molecular sciences underlying lubrication engineering. Currently, lubricant chemistry, especially "additive technology", looks like a very complicated field. It seems that scientific information is not always shared by researchers. The cause of this is that lubrication engineering is based on empirical methods and focuses on market requirements. In this regard, engineering knowhow is held by individuals and is not being disclosed to scientific communities. Under these circumstances, a bird's-eye view of lubricant chemistry in scientific words is necessary. The novelty of this review is to concisely explain the whole picture of additive technology in chemical terms. The roles and functions of additives as the leading actors in lubrication systems are highlighted within the scope of molecular science. First, I give an overview of the fundamental lubrication model and the role of lubricants in machine operations. The existing additives are categorized by the role and work mechanism in lubrication system. Examples of additives are shown with representative molecular structure. The second half of this review explains the scientific background of the lubrication engineering. It includes interactions of different components in lubrication systems. Finally, this review predicts the technical trends in lubricant chemistry and requirements in molecular science. This review does not aim to be a comprehensive chart or present manufacturing knowhow in lubrication engineering. References were carefully selected and cited to extract "the most common opinion" in lubricant chemistry and therefore many engineering articles were omitted for conciseness.
\end{abstract}

Keywords: tribology; tribo-chemistry; lubrication mechanism; lubricant formulation; molecular design

\section{Introduction}

\subsection{Tribology and Lubrication Engineering}

Tribology, defined as "the science of interacting surfaces in relative motion" [1], was coined in 1966 [2] by combination of "tribos" ("rubbing" in Greek) [1] + "logy". This term is becoming popular as we can find the term in dictionaries, not only in science and engineering fields but also in general culture. Although this name is relatively young compared to other engineering fields, empirical knowhow had been known from ancient days back to 2000 BC [3]. Now we can find various tribological phenomena in our daily life; dental care, shaving, wiping, cooking, etc. Needless to say, we enjoy modern civilized life using many machines; house equipment, vehicles, IT devices, industrial manufacturing, transportations, etc. We need tribology every time in everywhere. Thanks to lubrication engineering, we usually use these devices without being aware of tribology. However, we experience occasionally that the breakdown of a machine and this mostly happens due to failures of moving elements. This indicates the importance of lubrication engineering in prolonging the lifetime 
of machines. Lubrication also plays a significant role in improving energy efficiency. Moreover, lubrication engineering can contribute to comfortable machine operation by reducing unpleasant vibrations and noises that were developed from the moving contacts. Therefore, lubrication engineering does work hard in the background to support our civilized activities. Here, most readers of Applied Sciences would agree with the importance of lubrication engineering but might have little interest in it as research subject, probably because lubrication is a mature technology and hence is not a subject in applied sciences. Actually, lubrication engineering, especially lubricants, are still being developed by mostly trial and error procedures. So far, this has worked well for industrial applications. From now on, industrial R\&D has to pay attention to more energy efficiency with sustainability. Since conventional procedures consume time and energy, a smart method should be considered. In this regard, the author believes that the application of fundamental sciences in the right methods brings successful R\&D. Although many lubricants are manufactured by a combination of different substances, the understanding of the chemistry in lubricants seems rather underestimated. Actually, chemical information of lubricants is provided without detail and precise description, mainly due to the complexity of contents and the business matter. Although many tribologists state the importance of chemistry in tribology, they sometimes disregard "chemistry" because it looks like "a black box." This article surveys the chemistry of lubricant additives from viewpoint of fundamental chemistry, with the hope that it leads to a better understanding of tribological phenomena with molecular science. The author wishes the readers to be good supporters of tribology, especially those who are not familiar with machine elements. It would be an unexpected pleasure of the author if this article motivates a reader to undertake a tribology project with scientific aims.

\subsection{The Functions of Lubricants}

Lubricants are those substances that intervene between rubbing surfaces, thereby preventing any negative influences upon moving. The three major functions of lubricants are below.

- Controlling friction-This is the primary role of lubricants. Reducing friction and preventing wear and seizure (or failure) are necessary in most applications. Well-controlled, high friction is required for clutches, breaks, etc. Since this is the central objective of the lubricant, we discuss the phenomena with a lubrication model (Stribeck curve, see Figure 1). Optimized performances with minimized side effects could be achieved by proper selection of lubricants for specific machine elements. As modern machines are required to work in a more energy efficient way, the roles of lubricants are increasing. The importance of additives is emphasized (see Section 2.1 tribo-improvers).

- Cooling the contact-Heat generated by rubbing motion can have many negative influences on surface materials, such as transformation of microstructure or thermal failure. Ageing of lubricants is accelerated at higher temperatures. Heat accumulation could be prevented by circulating a liquid lubricant. The heat capacity of a lubricant is the controlling factor for this function. This function is mainly supported by the properties of base fluids. Some additives contribute to the heat conductivity of primary (see Section 2.5.2) or secondary (see Section 2.3.5) base fluids.

- Cleaning the contact-Wear particles, external dusts, or deposits by aged lubricants could appear during machine operation. These contaminants negatively influence lubrication performances. Circulating a lubricant can wash out these nuisances physically. Advanced lubricants contain some substances to help the cleaning process (see Sections 2.3.2 and 2.3.3).

Lubricants can appear as a liquid, as a semi-solid (greases), or as a solid (including particles and coatings) form. Since cleaning and cooling functions need fluidity, greases and solid lubricants could be applied to machine elements where heat and contamination are not serious factors. Because of the three functions and the benefit of handling, liquid lubricants are most ubiquitous in many practical applications. Therefore, various additives have been developed and are being applied in 
liquid lubricants. In this regard, this review mainly focuses on additives in liquid lubricants. Of course, the demands of greases and solid lubricants are also substantial mainly for task-specific purposes.

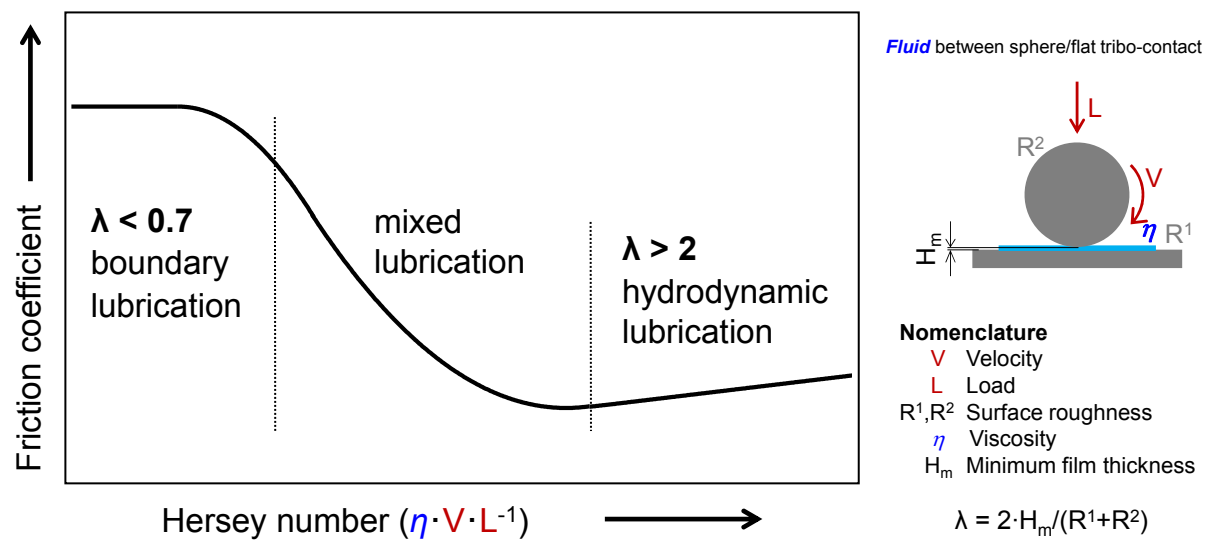

Note: $H_{m}$ (Minimum thickness of liquid film developed from base fluid between the surfaces, clearance of the surfaces) could be calculated from the operating parameters (load and velocity) and material parameters (elastic modulus and pressure-viscosity relations). For detail, see [5]

Figure 1. The Stribeck curve and lubrication regime.

\subsection{The Stribeck Lubrication Model}

There are many types of machine elements with different configurations that work under different operating parameters. Lubrication systems are individually designed in most cases. Despite this, we have a versatile model for simulation and explanation of the lubrication performances, the Stribeck curve [4]. The model concept is the dependence of friction coefficient on the operating parameters (load and velocity), the material properties (surface roughness and elastic modulus), and the lubricant properties (viscosity and pressure-viscosity relations). As Figure 1 depicts, the friction coefficient of a lubrication system varies with the Hersey number calculated from viscosity, load, and velocity. When a liquid (lubricating oil) is applied to tribo-contact, it develops a liquid film between the surfaces. The thickness of liquid film $H_{m}$ (or clearance between the surfaces supported by the liquid) depends both on the properties of the solid and the liquid materials [5]. A viscous liquid tends to provide a thicker liquid film at the contact, while a low viscous liquid tends to provide a thinner film between surfaces. If the thickness of the liquid film is greater than the surface roughness $R^{1}$ and $R^{1}$ (more precisely $\lambda>2$ ), the surfaces could be completely separated by the liquid. Therefore, the surfaces can move without contact between solids and this results in low friction. This lubrication regime is defined as "hydrodynamic lubrication". Ideally, machine elements are designed to operate in this regime since low friction as well as negligible wear could be achieved. When the Hersey number becomes smaller, the lubrication regime shifts to the boundary regime. Asperities on the surfaces come to interfere with each other. As a result, friction becomes higher and substantial wear occurs. This regime is defined as boundary lubrication. The properties of surfaces are important to achieve lubrication performances in this lubrication regime. Mixed lubrication regime is an intermediate regime between hydrodynamic lubrication and boundary lubrication. Although the phenomena in the real world are more complicated, this simplified model helps us to understand the role of each substance in lubrication systems. Today, the Stribeck model is a consensus model to predict the lubrication performance.

Relative importance of three functions varies with individual application. For example, metalworking fluids have to wash out cutting dusts from the contact and have to cool the tool surface simultaneously. High water based fluids are frequently used in metalworking applications. Although the lubricity is inferior to that of petroleum-based fluids, water-based fluids have strong advantages in cleaning and cooling functions together with cost effectiveness. Of course, the lubricity 
of metalworking fluid is necessary to prevent tool wear, for example, but cooling and cleaning are a technical priority in most cases. On the other hand, friction reduction is the major concern for bearing applications. This is why greases are mostly used in bearing lubrication where cooling and cleaning functions are of secondary importance. The differences in requirements of lubricants for different applications could be understood more clearly if we look at the Stribeck curve [4]. Bearings should be operated under hydrodynamic (precisely under elasto-hydrodynamic) conditions where wear of material and heat generation are trivial. On the other hand, metalworking processes are "heavily" influenced by boundary conditions.

When we look at the Stribeck curve carefully, friction increases as Hersey number increases under a hydrodynamic lubrication regime. This means that if the operating conditions are constant, friction increases as viscosity of the fluid increases. Since viscosity is defined as "a measure of the resistance to flow" [6], increased friction by viscosity could be understood as "internal friction in fluids". Anyhow, viscosity of fluids has to be controlled $t$ a proper level; that is as low as possible for achieving hydrodynamic lubrication. Additive technology for controlling viscosity is discussed in Section 2.2.1.

Although machine elements are designed to work under a hydrodynamic regime at steady state, they experience boundary regimes at least during start-stop operations where the velocity is 0 . Therefore, lubricants should support all lubrication regimes, from hydrodynamic to boundary lubrications, and hence different functions are required. This is the main reason for the complexity of the lubricant in which different additives are required in a lubricating fluid. Thanks to successful research by both experiments and simulations, the Stribeck curve well represents the phenomena under (elasto-)hydrodynamic regimes. In general, we can use lower viscosity lubricants for fast-running machines in comparison with slow-running machines. Similarly, heavy-load machines need higher viscosity lubricants, compared to light-load machines.

Wear of tribo-material happens under boundary and mixed lubrication regimes where direct surface-surface contact occurs. This leads to changes in surface roughness and a possible shift in lubrication regimes as a consequence. However, the Stribeck curve is based on the assumption that the material parameter (surface roughness in this case) is constant. It should be noted that the Stribeck curve does not fully support for boundary and mixed lubrication regimes. Nonetheless, it indicates inferior lubrication performances under boundary and mixed lubrication regimes compared to those under hydrodynamic lubrication.

\subsection{Tribo-Chemical Reactions}

Mechano-chemical reactions have a long history from the alchemist ages [7-10]. Since then, various chemical reactions have been empirically examined under mechanical stresses such as agitation in pestle-mortar or collision of solids. In addition to scientific approaches [11,12], industrial applications of mechano-chemical reaction could be found in preparation of materials $[13,14]$ and waste treatments [15,16]. The term, "tribolysis" was first published in 1928 [17]. It seems to indicate "tribo-chemical reaction" but this article is describing alterations of petroleum products by mechanical agitation (traditional tool for mechano-chemical reactions) compared with photolysis and thermolysis of these substances.

Tribo-chemical reactions could be categorized as a part of mechano-chemical reactions. $t$ should be defined precisely as "conversion of molecules to others under the influence of rubbing". Additionally, this review focuses on this phenomenon "especially under lubricated conditions". In this regard, chemical reactions of lubricants, both base fluids and additives, are of interest. It is generally agreed that tribo-chemical reactions are complicated phenomena. There are two major causes of the complexity. One is the problem with the analytes. Lubricants are mixture of various compounds, including base fluids and different additives. In most cases, their principle structure is hydrocarbons and hence clear identification of each substance is difficult. When we focus on the reaction mechanism of an additive of interest, the problem of the detection limit with instrumental analysis usually arises. Concentration of additive could be as low as in the range of mmol $\mathrm{kg}^{-1}$. Specific analytical tools, such as surface 
sensitive analysis, combined with a model lubricant (e.g., a solution of single additive in a solvent) could be a solution for the analyte problem. Actually, many articles are being published on the mechanism of tribo-improvers (see Section 2.1) with this procedure.

On the other hand, much progress in understanding of the energy for reaction is necessary. Chemical reaction is an arrangement of atoms within or between molecule(s), and is accompanied with breaking and making chemical bonds. This process needs energy to occur; tribo-chemical reaction is a conversion of mechanical energy into chemical energy in one sense. From this point, possible driving forces (sources of energy for chemical reactions) for tribo-chemical reactions are summarized in Figure 2. Six possible driving forces are considered for the initiation process of tribo-chemical reactions [18].

- Heat induces various chemical reactions. It was estimated that rubbing surface could reach as high as $250-450{ }^{\circ} \mathrm{C}$ [19]. Friction generates heat and this is considered as the major cause of tribo-chemical reactions.

- Nascent surfaces could be exposed by wear of solid surfaces under mixed and boundary conditions. Similarly, a mechanical stress to crystalline materials can sometimes cause lattice defects. These produce a chemically active site on the rubbing surfaces [20]. The chemical activity of transition metals is induced by vacant d-atomic orbital.

- Exo-electrons could be emitted from rubbing surfaces. Those electrons have low energy to promote chemical reactions but can produce radical intermediates for further chemical reactions [21].

- Elevated pressure up to Giga-Pascal ( $>10^{4}$ bar) could be generated at tribo-contact [22]. Since chemical reactions are initiated by a collision of molecules, compression of reactant raises the probability of the collision. Chemical reactions at high pressure under static conditions are well known [23].

- Orientation of molecules may occur if they were flown through a narrow area [24]. This means the reacting functional groups are close to each other and increases the probability of reaction. This, together with elevated pressure, contributes to the entropy factor of the reaction.

- Shearing can dissociate chemical bonds in a molecule directly. It is a well-known phenomenon that molecular mass of polymers can decrease under shearing conditions. The formation of radical species by the dissociation of a carbon-carbon bond had been reported [25]. Typical shear rate at tribological contact is in the range of $10^{5}-10^{7} \mathrm{~s}^{-1}$ [19].

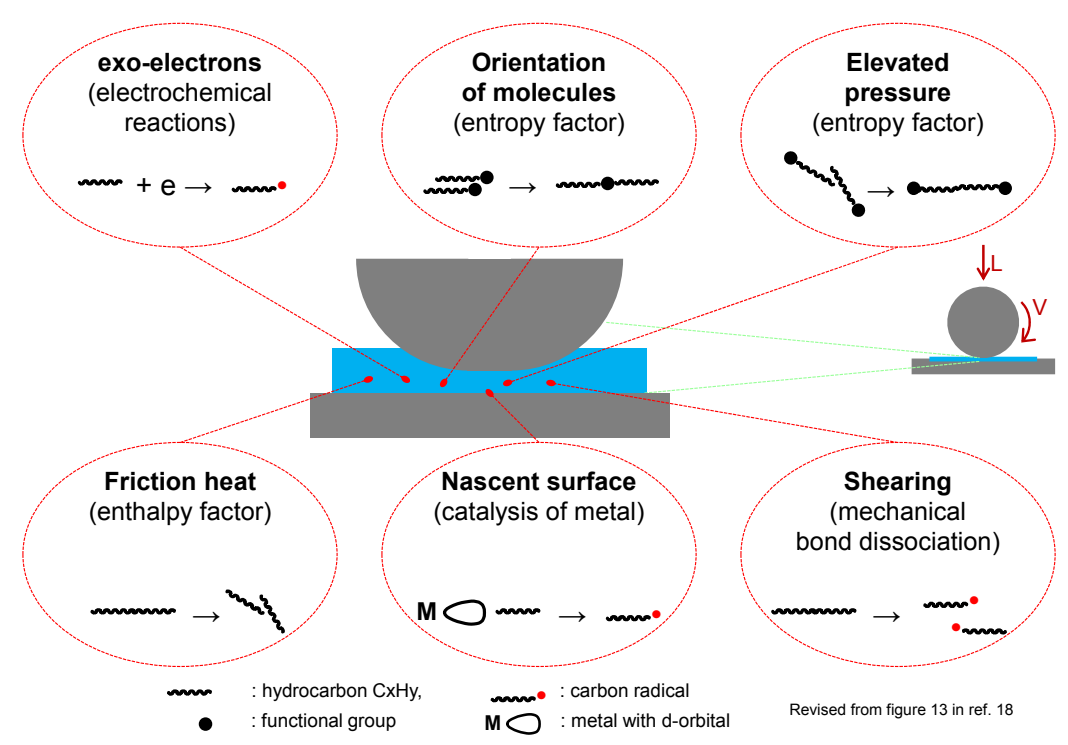

Figure 2. Possible driving forces for chemical reactions at tribo-contact. 
A tribo-chemical reaction can beneficially or detrimentally influence to the lubrication performance. There is a consensus in the tribology community that tribo-chemical reactions concern mainly boundary lubrication conditions. However, from the viewpoint of a reaction mechanism, they can occur under (elasto-)hydrodynamic conditions as well; elevated pressure, heat by compression, and shearing are three examples.

\subsection{Components in Liquid Lubricants}

So-called lubricant formulation is a process of mixing substances to prepare a lubricant. The substances should be properly selected to meet the requirements of machine operation. The components in liquid lubricants are classified in two groups-base fluids and additives. Base oils are the major contents of lubricating fluids. It has been empirically known from ancient times that viscous fluids provide lubrication performances. It was found, about a hundred years ago, that certain substances can improve lubrication performances if dissolved in base fluids [26]. Then many engineering knowledge was accumulated and additive technology for lubricant became a common technique later on. Today, lubricating fluids are manufactured from base oils and 5-20 mass\% of different additives depending on the practical requirement (Graphic abstract). Base fluids are viscous liquids having proper viscosity for the application. API defines the category of base oil, as summarized in Table 1. API Groups I-III are made from crude oil through distillation and refinery processes [27]. They are so-called mineral oils and they constitute the majority of base fluids today because of their availability at a reasonable cost. In brief, mineral oils are composed of various hydrocarbons (normal and branched alkanes, cycloalkanes, aromatics, heterocyclic compounds, etc.). It seems difficult to show the chemical contents in mineral oils, because they vary with production area and refinery processes. Therefore, viscosity represents the properties of base oil. Conventionally, kinematic viscosity at $40{ }^{\circ} \mathrm{C}$ and at $100{ }^{\circ} \mathrm{C}$ is considered for lubricants. The refinery processes have two major purposes; they are purification and modification of molecular structure. Typically, organic sulfides and unsaturated hydrocarbons were extracted by solvents to produce Group I, or decomposed by a catalytic hydrogenation to produce Group II. The importance of these processes for additive technology is that these impurities often show antagonism to many types of lubricant additives. On the other hand, a catalytic isomerization of hydrocarbon molecules produces Group III oils. The differences between Groups I-II and Group III oils are their rheological properties.

Here we introduce the key properties of base fluids; viscosity index: dependency of viscosity by temperature. Viscosity of hydrocarbons drops as temperature increases. Viscosity index (VI) represents the rate of changes by temperature [28]. "High" viscosity index is defined as fewer changes in viscosity by temperature change, as illustrated in Figure 3. Both fluid A and B pose similar viscosity at $40{ }^{\circ} \mathrm{C}$, while those at $100{ }^{\circ} \mathrm{C}$ are different. Needless to say, higher $V I$ is desirable because many lubrication systems generate friction heat during machine operations. When considerable viscosity drop happens during machine operation, it causes a shift of the lubrication regime to boundary (see Figure 1). As a consequence, it raises the risk of high friction and wear. We also discuss this issue in Section 2.2.1. VI could be calculated according to ASTM D2270, for those fluids of VI <100. It is recommended to use software on computers, since currently base fluids with $V I>100$ are commonly used [29].

Besides mineral oils being the major resources of base fluids, natural triglycerides (TG, also known as vegetable oils, plant oils, or animal tallow) were the only resources of lubricants before the petroleum age. Although they were used before petroleum products, TGs are currently classified in Group V because of the fewer demands compared to mineral oils. Technically, those additives for mineral oils do not always work well for TGs. One reason for this is that additives are manufactured by petroleum industries. Therefore, industries are interested in improving the performances of petroleum products. The relevant scientific discussions are given in Section 4.4. 
Table 1. API base oil category and examples.

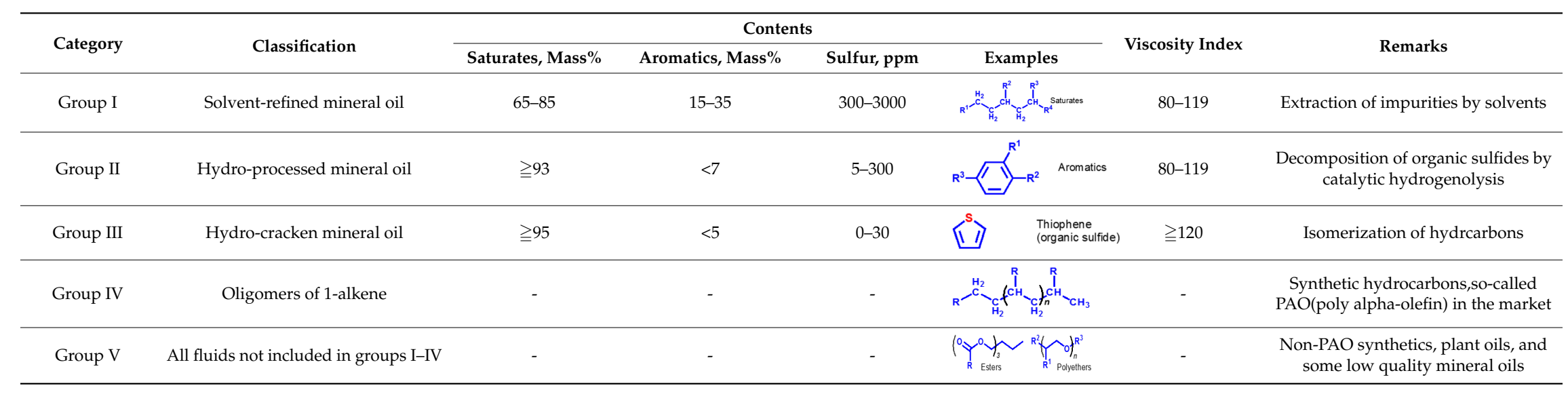




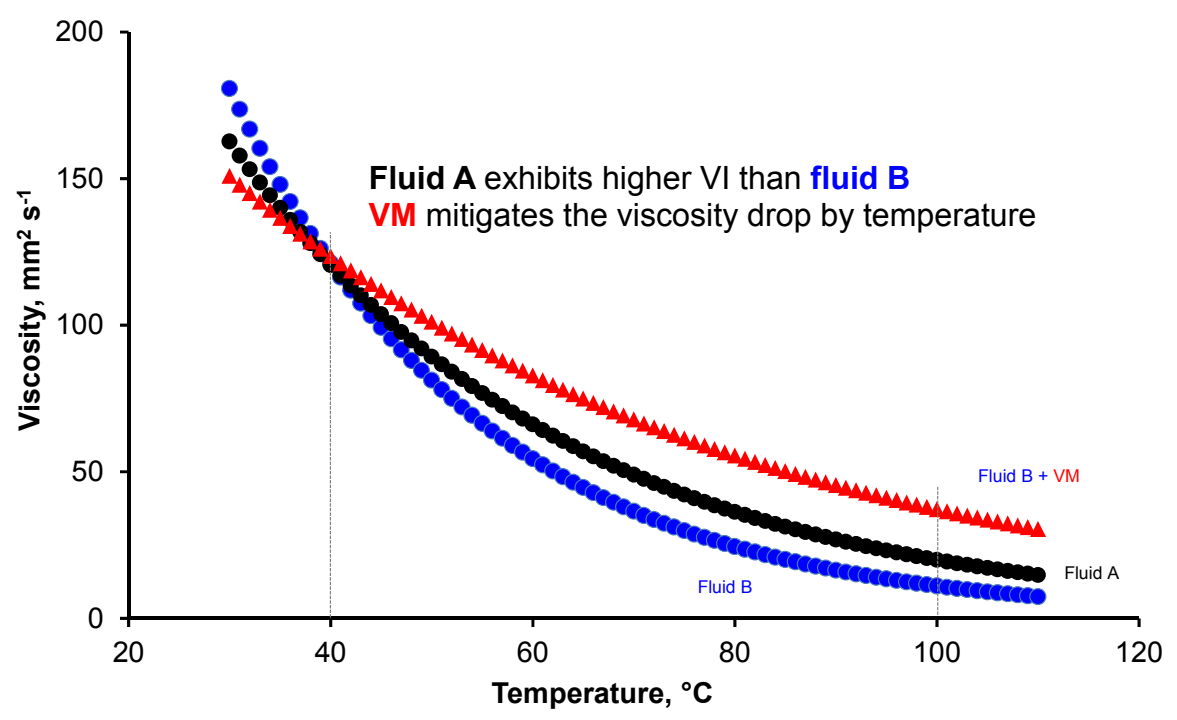

Figure 3. Temperature-viscosity relation and viscosity index.

In addition to MOs and TGs, there is growing demand for base fluids prepared artificially, so-called synthetic fluids (SF). Huge types of SFs have been introduced and are being developed for lubricants. Among them, synthetic hydrocarbons prepared by the oligomerization of 1-aklens form an individual category, Group IV. They are called "poly-alpha-olefin (PAO)" as a market code. This pseudo-chemical naming is an example that makes chemists confused.

Other synthetic hydrocarbons, synthetic esters, and poly-ethers are also being used as base fluids. The importance of SF is, in contrast to MOs and TGs, the possibility of molecular design for various purposes [30]. Similar to the issue with TGs, conventional additive for MOs cannot always be applied to SFs, as discussed in Section 4.4.

Although the concept of molecular design can create molecules with required properties as lubricants, it is difficult to support all requirements for a lubrication system with base fluid alone in a cost effective manner. Here we shall recognize the importance of task-specific lubricant additives. They are defined as those substances that improve the total performances if added to base fluids properly. The performances include both tribological and material related ones. The former contribute to reducing friction and preventing wear and seizure during machine operation. The latter mainly contribute to the lifetime of the lubricant and/or tribo-materials. The method of improvements is ideal to enhance the advantages of base fluids with a synergistic effect. In the real world, additives make up for the shortages in specific properties of base fluids in most cases. Additives as liquid forms have advantages of handling in liquid lubricants. Solid additives, either as solution or as suspension, are also commonly used. Greases could be used in some cases.

The application of lubricant additive is relatively recent in the long history of lubrication engineering. To the best of the author's knowledge, the first publication of the phenomena in a scientific journal was nearly 100 years ago [31]. According to the publication, lubricant additives have been practiced in the market since the 1950s [26]. Since then, lubricant additives have been developed by engineering knowhow. This might be the main reason why the descriptions in this field are less-scientific; confusing terminology, unclear chemical structure, complexity of contents, etc.

\subsection{Category of Lubricant Additives}

Many types of lubricant additives can be found in the market. In most cases, they are being sold according to their main roles in lubrication systems, such as anti-wear or anti-oxidation agents, and friction modifiers. It would be instructive to classify those substances according to their working functions, the means of action (how does it work), and their working site. 


\subsubsection{Working Function Category}

This is an upgraded categorization of existing methods, grouping "substances by their role" into large groups. All additives belong to one of the following groups.

- Tribo-improvers (tribology-improving additives) directly contribute to improve the tribological performances of the lubricant. In short, they are responsible for the primary role of lubricants (see Section 1.2). Friction modifiers (FM), anti-wear agents (AW), extreme pressure additives (EP) are representative ones. They stand at a central position in the technology of lubricant additives.

- Rheo-improvers (rheology-improving additives) concern the fluidity of the base oil. Viscosity modifiers (VM) are the main additives in this group. Pour point depressants (PPD) make the base oil applicable in a chilly environment. They indirectly contribute to the lubrication performances, mainly in hydrodynamic regime.

- Maintainers help to keep the substances (both lubricant and materials of machine elements) in good condition through preventing the degradation of substances participating in the lubrication system. They mainly contribute to prolonging the lifetime of the lubrication system and partly contribute to the lubrication performances in some cases. Antioxidants $(\mathrm{AO})$ play a decisive role in preventing the ageing process of lubricants. Detergents and dispersants can mitigate the negative influences of contaminants on lubrication. Corrosion inhibitors (rust preventives) can protect the tribo-materials from corrosion. Air bubbles may be incorporated in lubricants during machine operation. They cause lubricant starvation at the contact and promote the autoxidation processes. Anti-foam agents (foam breaking agents, defoamers) can break bubbles. Water is a ubiquitous contaminant in most applications. It drops the viscosity of the lubricant and causes ageing of both lubricants and materials. Demulsifiers (emulsion breaking agents) are beneficial for separating contaminated water in a lubricant.

- Auxiliaries are sometimes incorporated with specific purpose in addition to above additives.

\subsubsection{Working Site Category}

From another viewpoint, the working site of each additive can be characterized, as below. This category focuses on the action mechanism (the way the additive works) at a molecular scale rather than practical performances.

- Interface agents work at the interfaces between different phases. Lubricated contacts involve solid (tribological material)—liquid (lubricant) interfaces. Examples are tribo-improvers as the main actors in this category. Corrosion inhibitors deactivate the surfaces to be attacked by corrosive matters. A lubricant may intake air bubbles while circulating, and thereby develops various forms. The interaction of foam decomposing additives at the border between gas-liquid phases can destroy the bubbles.

- Bulk agents concern the properties and/or stability of liquid as uni-phase matter. Rheo-improvers are representative examples of this group. Antioxidants (except metal deactivators), and other auxiliaries are in this group.

\subsubsection{Working Mechanism Category}

This gives more insight into the action mechanism, how a molecule behaves and/or changes while working. It also connects to the level of energy to activate the functions.

- Chemical additives are those additives that undergo chemical reaction(s) while working. A chemical reaction is defined as a rearrangement of electrons that bind atoms in a molecule. Chemical changes at interfacial phenomena in tribology are mostly irreversible, although there is a certain possibility of reversible reactions in the liquid phase. The initiation of chemical reaction needs much more energy than physical phenomena. Examples are anti-wear agents that provide 
boundary film on rubbing surfaces through tribo-chemical reactions. Antioxidants also belong to this group.

- Physical additives are those substances that work without any chemical changes. Examples are nano-particle additives such as tribo-improvers and viscosity modifiers. Physical changes need less activation energy than chemical changes and are usually reversible processes. They last longer than the chemical ones.

The above categories are independent of each other. The function category is a universal criterion mainly for engineering applications and overviewing the technology. Phase and scientific categories focus on investigation of mechanism and developing new technologies through molecular design. Table 2 depicts an interactive character of these categories for individual lubricant additives.

Table 2. Relations of different classification of additives.

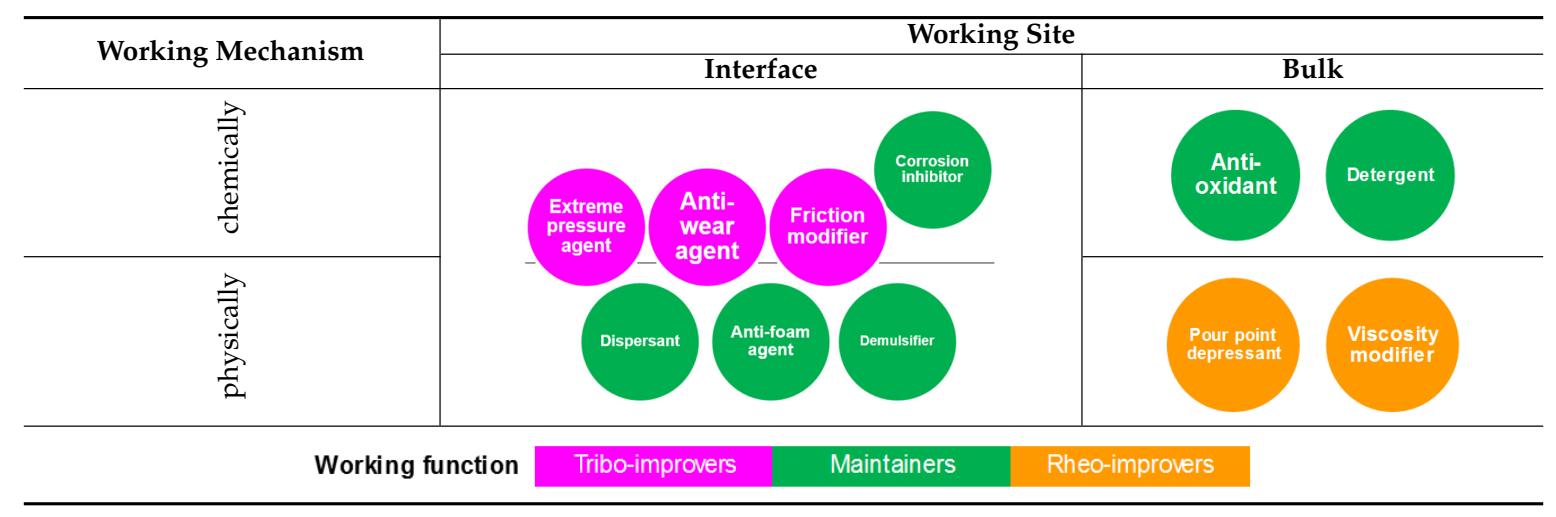

\section{Individual Lubricant Additives and Their Working Mechanism}

This chapter introduces individual additive categories related to the role in lubrication. Although the description follows conventional ways to introduce lubricants [32-34], this chapter primarily focuses on how molecules behave for lubrication performances. Representative molecular structure and the process of action are given briefly to help the readers' understanding and further scientific investigations. The science of lubricant additives, especially their action mechanism, is under discussion in the tribology community and is still under investigation by different researchers. Here the author tried to show the consensus mechanism, as the "greatest common measure" extracted from carefully selected articles.

\subsection{Tribo-Improvers}

Those additives contribute to the primary purpose of a lubricant and many R\&D activities have been and are being performed intensively. Therefore, this review puts special emphasis on this category. There is a consensus among tribologists and lubrication engineers that tribo-improvers could be conveniently classified into FMs, AWs, and EPs. Generally, FMs effectively work for "relatively light-load" mixed lubrication while AWs prevent wear for "harder" mixed lubrication, depicted as a simple Stribeck curve in Figure 4. It is empirically known that their effects overlap each other. That is, friction reduction by FM is usually associated with wear reduction to some extent. Similarly, AW primary prevents wear and sometimes reduces friction, but not always. In consequence, the demarcation between FM and AW is not clear. This can be understood by their working mechanism below. 


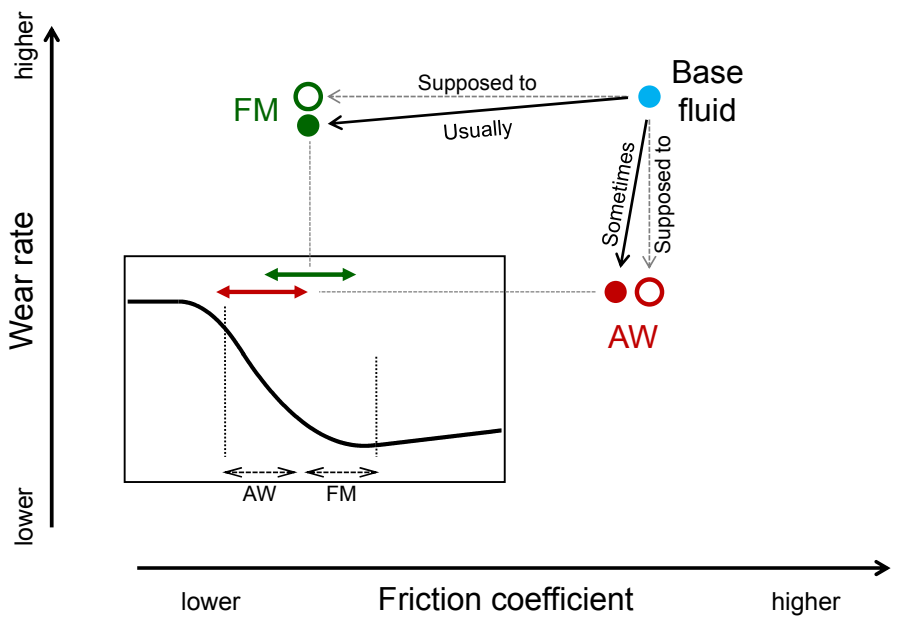

Figure 4. Overlap effects of tribo-improvers.

\subsubsection{Friction Modifiers (FM)}

Historically, carboxylic acids with a straight hydrocarbon chin (higher fatty acids) were the first recognized "lubricity improving substances" when they were dissolved in mineral oils [35]. They have been well-studied from both a scientific and engineering perspective in the past 70 years. The benefit of carboxylic acids in scientific research is the availability of pure compounds as commercially available reagents, although their practical applications are trivial [36]. However, an analogous molecular structure could be found in various practical additives, indicating the importance of good understanding in the working mechanism from a scientific viewpoint.

Figure 5 explains the effective working mechanism of carboxylic acids (octadecanoic acid, $n-\mathrm{C}_{17} \mathrm{H}_{35} \mathrm{COOH}$ is the most frequently employed in fundamental research) for steel as a tribo-material. Engineering surfaces are usually covered with contaminants or oxide layers, which can be removed by rubbing. This exposes active surfaces that interact with the FM molecule. Further rubbing provides the energy to the molecule for surface reactions. It produces organic salt (salt of carboxylic acid), and results in chemisorption (adsorption with chemical bond between adsorbent and adsorbant). Chemisorption possesses a strong interaction with the surface compared to that of physisorption [37], and hence it is beneficial for protecting the film against rubbing [38]. The alkyl group in the molecule has significant role in lubrication. Direct asperities, contact upon rubbing could be mitigated by the adsorbed FM molecules. The Van der Waals attraction force could be generated between oriented molecules. It strengthens the robustness of the film to support the load applied to the contact [35]. In fact, alkyl chains having $>12$ methylene (- $\mathrm{CH}_{2}$ - unit) are beneficial for boundary lubrication [39]. This model has been proved under dynamic conditions (so-called "in lubro") using optical interferometry [40] or liquid cell scanning probe microscope [41].

Although the interaction of the acid moiety with the surface is a chemical process, the hydrocarbon group (commonly straight hydrocarbons having 15-17 carbons), a major part of the molecule, is supposed to be unreacted. However, common spectroscopy cannot provide clear evidence of additive molecules that are mainly composed of the hydrocarbon moiety. It should be noted that the concentration of FM is usually around $10 \mathrm{mmol} \cdot \mathrm{kg}^{-1}$ in hydrocarbons as base fluids and hence it is difficult to identify additive molecules as highly diluted solutions. Nonetheless, clear evidence of chemisorption was obtained by surface mass spectroscopy using deuterium-labeled molecules as model FM [42]. This is frequently misunderstood by tribologists as "those FMs physisorp on surfaces", probably because the alkyl group is unreacted. A term "quasi-physical adsorption" might be convenient to distinguish those FMs from "tribo-chemical types" explained below.

Some lubricant additives take advantage of tribo-chemical reactions. Figure 6 illustrates the working mechanism of organic salts of molybdate. The first step is the interaction of additive molecules 
with the rubbing surface, which is an analogous mechanism in Figure 5. The difference appears in the next step. When the tribo-process provides enough energy to the molecule, the molecule decomposes to inorganic salts. Since the reaction is associated with the dissociation of many chemical bonds, it needs relatively higher energy than the chemisorption process. The inorganic salts (molybdenum disulfide as the example in Figure 6) are usually more robust than organic salts to heat and mechanical shearing. Contrary to the chemisorption mechanism, the organic moieties in an additive molecule were decomposed during the tribo-film formation. In this regard, the main role of the organic moiety is to dissolve the precursor of inorganic salt in base fluids (mostly hydrocarbons). Some contributions of carbon-deposit to the tribological properties have been also suggested for the tribo-chemically derived film [43].

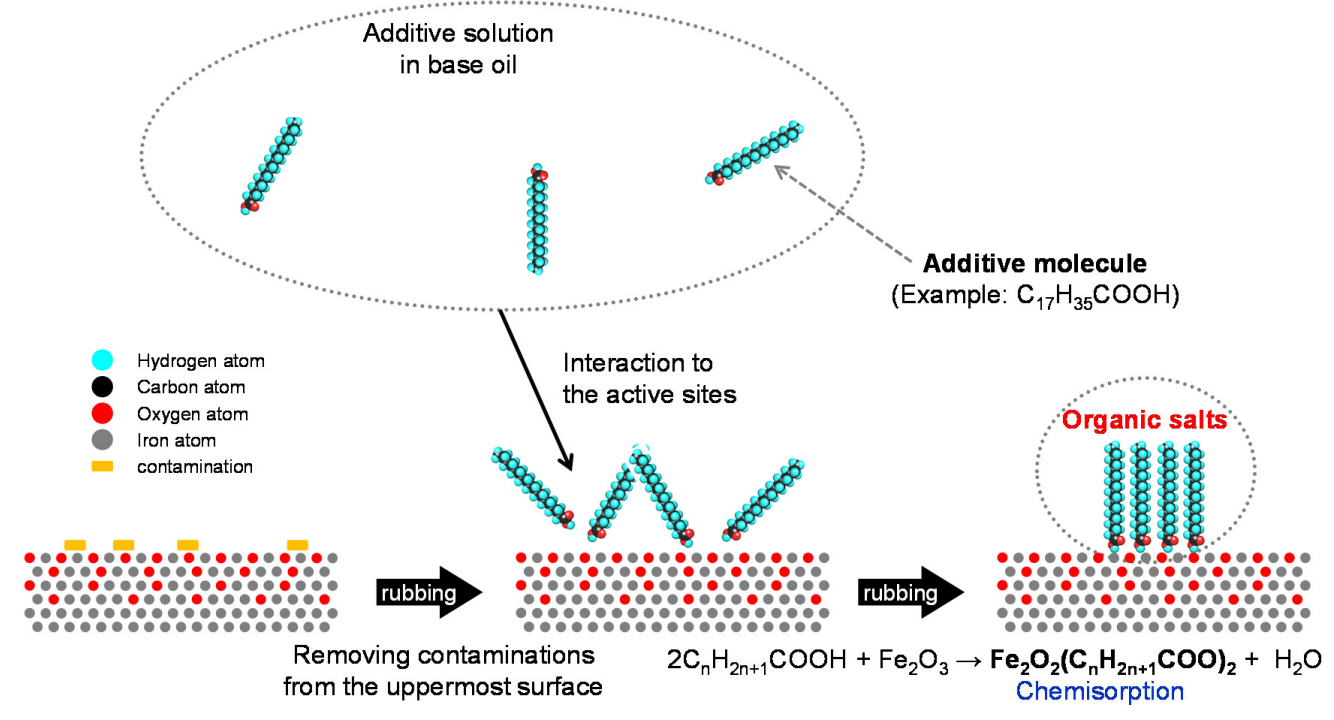

Figure 5. Working mechanism of adsorption type tribo-improvers.

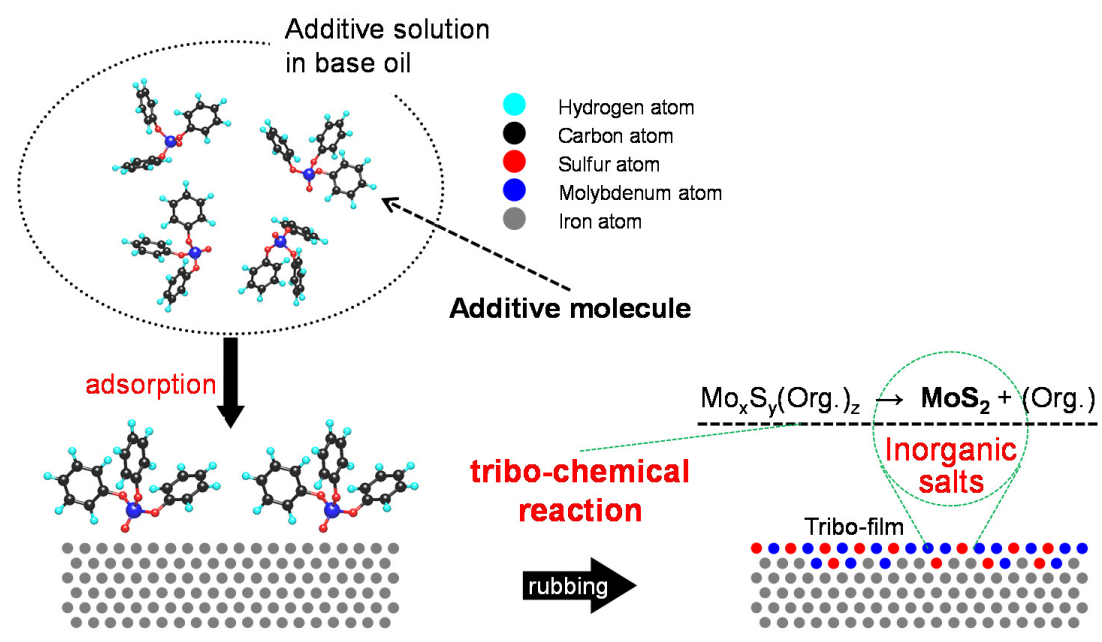

Examples of tribo-chemical reaction for AW

$$
\left\{\begin{array}{l}
\mathrm{Zn}\left[(\text { Org. })_{2} \mathrm{PS}_{2}\right]_{2}+\mathrm{Fe}_{2} \mathrm{O}_{3} \rightarrow \mathrm{Fe}_{\mathrm{x}} \mathrm{Zn}_{\mathrm{y}}\left[\mathrm{O}\left(\mathrm{PO}_{2}\right)_{z} \mathrm{O}\right]_{\mathrm{a}}+(\mathrm{Org} .) \\
\mathrm{P}_{\mathrm{x}}(\mathrm{Org})_{\mathrm{y}}+\mathrm{Fe}_{2} \mathrm{O}_{3} \rightarrow \mathrm{FePO}_{4}+(\mathrm{Org} \text {. })
\end{array}\right.
$$

Where Org. $=\mathrm{C}_{x} \mathrm{H}_{y} \mathrm{O}_{z} \mathrm{~N}_{\alpha}$

Figure 6. Working mechanism of tribo-chemical reaction type tribo-improvers. 
Molybdenum disulfides are well-known solid lubricants [44]. Certain oil-soluble molybdenum salts are practical FMs [45-47] as precursors of molybdenum disulfide.

Inorganic particles, such as soft metals or carbon allotropes, can physically interact with the rubbing surfaces and thereby improve the tribological properties [48-52]. They could be used not only for FMs but also AWs or EPs depending on their properties. Their stable dispersion has to be prepared for liquid lubricants. The details are omitted because this review pays much attention to molecular structure.

Some task-specific tribo-improvers have been developed. For example, the importance of "anti-shudder agent" is sometimes pointed out for driveline systems. It prevents stick-slip due to higher static friction than dynamic friction, which results in the vibration of material being in the same direction as the motion. This belongs to FM and analogous substances to FMs are employed.

\subsubsection{Anti-Wear Agents (AW)}

Wear is a change of shape on surfaces through eliminating a small portion of the material from the triboogical contact. It happens under relatively hard mixed lubrication conditions where adsorption type FMs cannot support the lubrication performances. As discussed in the mechanism of reaction-type FMs, inorganic salts generated by tribo-chemical reactions are suitable. The differences between AWs and reaction-type FMs are not the chemical process of tribo-film formation but the physical properties of the tribo-film provided at the tribo-contact. In general, hard materials are more robust to wear compared to soft material. Inorganic phosphates on steel surfaces are well known as the protecting film [53]. Zinc bis(dialkyldithiophosphates) (ZnDTPs) are the most widely used AWs in practical lubricants so far [54]. Intensive studies on working mechanism of ZnDPTs by surface analysis have revealed the formation of inorganic compounds composed of phosphorus, sulfur, and zinc [55]. The process of boundary film formation has been studied experimentally [56-59] and in-silico [60]. Co-existence of other tribo-improvers such as molybdenum carbamates displays improvements in lubrication performances [61-64]. Organic esters of phosphoric and phosphonic acids also display AW properties for steel surfaces [19].

\subsubsection{Extreme Pressure Additives (EP)}

Formerly, these were so-called as load carrying agents. When the tribological conditions become severe, at higher load and/or higher temperature, welding of the material may occur. EP can prevent such failure of materials upon rubbing contact. Their functions are unique compared to FMs and AWs. EPs first react with the surface, which is analogous to AWs. However, the product does not protect the surface but mildly removes the atoms on the uppermost surfaces (Figure 7). The reaction of an EP molecule (dialkyl disulfide in this case) with the atom $-\alpha$ on the surface is associated with migration of a bonded-electron from the material, as depicted by a red arrow. It weakens the interaction of the atom $-\alpha$ with other atoms in the material resulting facile migration of atom $-\alpha$ from the material. The wear process releases the mechanical energy at the contact point, otherwise the accumulated energy can induce serious damages to the tribo-materials. The function is so-called "controlled wear mechanism". This is closely related to the mechanism of "corrosive wear" or "chemical wear" where certain chemical substances promote the wear of material. Contrary to the other wear processes such as abrasive and adhesive wear, corrosive wear produces relatively smooth surfaces (less surface roughness). Chemically aggressive substances can induce corrosive wear. Similarly, EPs are relatively reactive with surfaces are compared to substances for FMs and AWs [65].

It is empirically known that EPs can help the running process in an efficient way. This could be understood by the smooth removing of the initial asperity on the surfaces. In some cases, EPs can provide "rescue tasks" when the machine elements are under fatal conditions with severe wear. EPs are expected to lower the contact stress through the corrosive wear. This can make the lubrication regime milder. However, the effect should not last. 


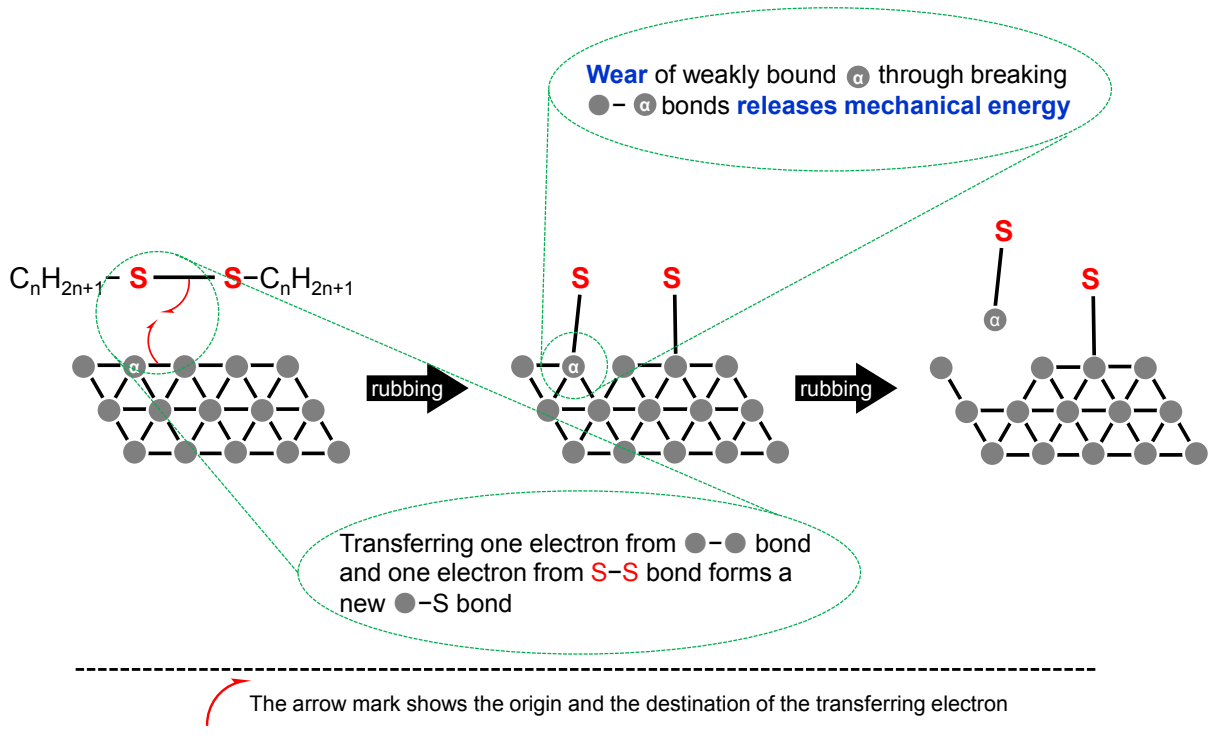

Figure 7. Working mechanism of EP (controlled wear).

Contrary to the extensive academic researches on FMs and AWs, scientific approaches to EP functions are still fewer, mainly due to the difficulties of analysis. Evaluation of EP such as ASTM D2783 results in welded surfaces with relatively poor repeatability.

In summary, all tribo-improvers work on the surface under dynamic processes. In other words, the mechanical energy continuously stresses the rubbing contact. It damages the surface more or less, resulting in wear, for example. Here, the importance of recovering the tribo-film has arisen, as pointed out in Figure 8. As discussed supra, the function of all tribo-improvers start with the interaction to the rubbing surface. Then the intermediate material (the transition state) is converted to the tribo-film through adsorption or tribo-chemical reaction, according to the lubrication conditions. When the machine works under high wear rate conditions, the regeneration process should be done quickly. An increase in affinity of the additive to tribo-materials, or an increase in reactivity under the tribological conditions is the usual method of lubricant chemistry. An increase in robustness of the tribo-film is challenging. Multi-functional tribo-improvers are possible by a proper combination of each moiety in FM, AW, and EP molecules (Figure 9).

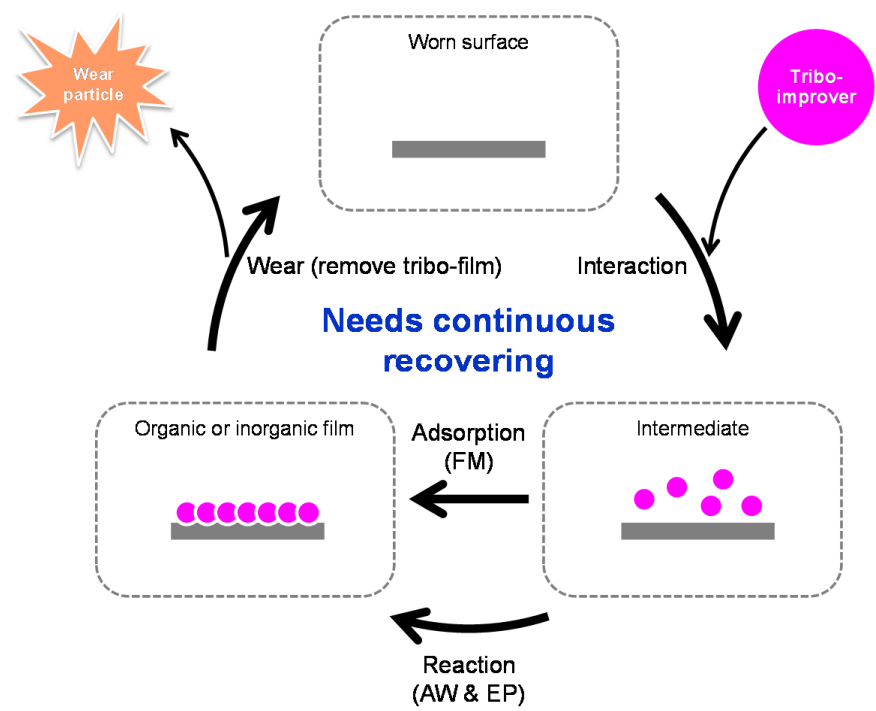

Figure 8. The importance of dynamic process for tribo-improvers. 


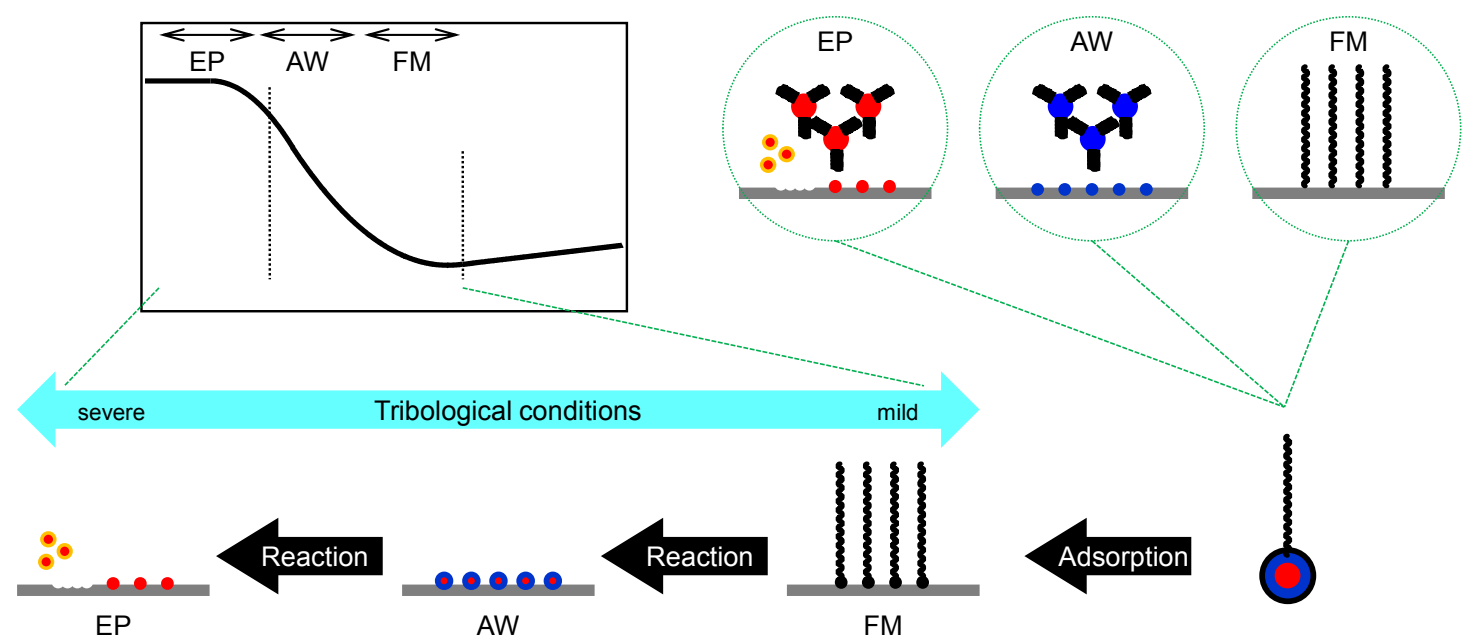

Figure 9. Multi-functional tribo-improver (model).

\subsection{Rheo-Improvers (Rheological Properties Improvers)}

Viscosity is the primary property of base fluids for liquid lubricants, as discussed in Section 1.1. Those substances that are responsible for fluidity of base oil are rheological property improvers. They contribute to the lubrication performance by modifying the bulk properties of the liquid, mainly under hydrodynamic conditions. From the viewpoint of functions, rheo-improvers could be categorized as "indirect" tribo-improvers between mixed and hydrodynamic lubrication regimes.

\subsubsection{Viscosity Modifiers (VM)}

Formerly, VM were called viscosity index improvers (VII). The retired name indicates the role of the additive more directly. VI (see Figure 2 and Section 1.5) is an engineering parameter to qualify the lubricants in practice. The bulk properties are dependent on the structure of the base fluids. In fact, the manufacturing process of the API Group III includes the isomerization of hydrocarbons. Structural features of VM are polymers [66]. The molecule can be compactly packed at lower temperatures, while it expands at higher temperatures (Figure 10). It is expected that molecules with large volume possess resistance to flow, thereby displaying high viscosity [33]. Applicable temperature range of lubricants was made wider by improving VMs. A technical challenge for advanced VMs is stability under shearing (see Figure 3 and Section 1.4) [67].

- Base fluid molecule

Principle chain of VM molecule

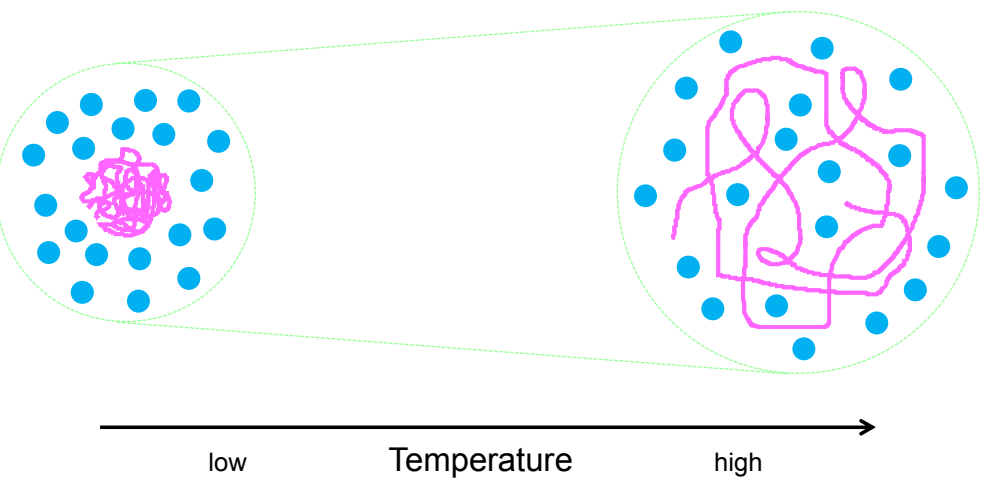

Figure 10. Working mechanism of viscosity modifier. 


\subsubsection{Pour Point Depressant (PPD)}

Lubricants may freeze if while machine pauses at low temperatures. This causes lubrication failure and leads to serious damages to machine elements. A pre-heating system can prevent the problem, but it consumes considerable time and energy. PPDs are beneficial for machineries under cold climates. PPDs are typically branched hydrocarbons [68]. Each branched alkyl group can interact with oil molecules, thereby preventing the crystallization of the oil molecules together. Because of the branching structure, the PPD molecule can keep free volume around the molecule that this makes the partial structure mobile (Figure 11).

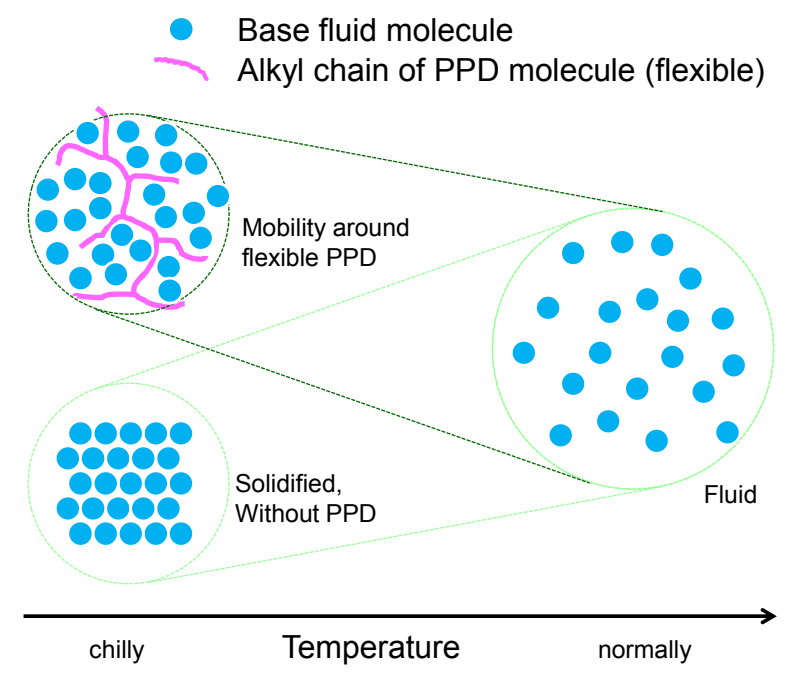

Figure 11. Working mechanism of pour point depressant.

Both VM and PPD are macro-molecules (polymers) compared to the molecular mass of base oil molecule. However, the functions of VM and PPD are different. The main function of VM is to suppress mobility at higher temperature thereby increasing the viscosity.

On the other hand, branched structure is beneficial for PPDs because the molecule has to be responsible for mobility at lower temperatures. The functions of both additives involve a physical effect and the changes are reversible by temperature change unless the molecule decomposes.

\subsection{Maintainers}

Tribology is an entropy-increasing process that generates heat and worn particles as mechanical energies are lost. It is usually accompanied with retarding of lubricants and tribo-materials, resulting in inferior lubrication performances called "lubricant ageing". Maintainers mitigate any negative influences on the lubrication performance. In most cases, the ageing processes are accelerated by internal and external contaminants accumulated in the system while the machine works. The function of maintainers is either pro-active or post-active.

\subsubsection{Antioxidants (AO)}

Autoxidation is the oxidative degradation of organic compounds that occurs spontaneously under aerobic conditions without any active treatments such as reagents, catalysts, or driving forces. Most organic materials experience this reaction as ageing processes, and lubricants are no exceptions. Therefore, they have been well studied both from engineering and scientific approaches [69-73]. The consensus reaction pathway is the radical chain reaction mechanism, which is initiated by the hemolytic dissociation of a $\mathrm{C}-\mathrm{H}$ bond by heat or ultraviolet irradiation. Figure 12 explains the possible mechanism of radical formation under tribo-chemical conditions.

- Thermal dissociation of a carbon-hydrogen bond to yield hydrogen radical and carbon radical. 
- Shear stress can dissociate a carbon-carbon bond though direct mechanical forces, yielding two carbon radical intermediates. Polymers can decompose through this mechanism.

- Wear of tribo-materials exposes nascent surfaces that may catalyze the dissociation of a carbon-hydrogen bond.

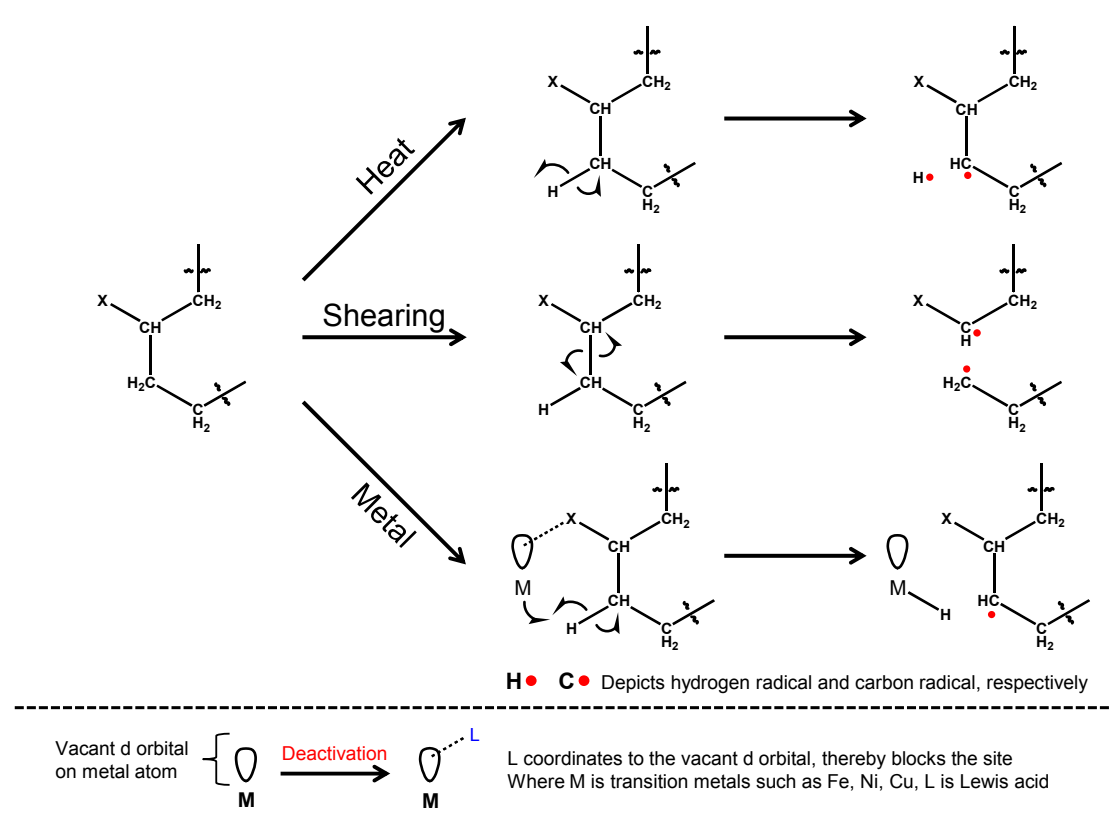

Figure 12. Initiation step of autoxidation (generation of radical intermediates).

Among the three initiation mechanisms, causes by shearing and nascent surfaces are unique in lubrication systems (see Section 1.4). The carbon radicals are ready to react with oxygen to yield peroxy-radical species that further react with another hydrocarbon molecule (Figure 13). The reaction results in regeneration of the carbon radical intermediate and oxidized molecule. This regeneration step is the characteristic to the radical chain reaction mechanism. Theoretically, the autoxidation can proceed again and again until the depletion of the hydrocarbon once the carbon radical species were generated. It should be noted that the radical intermediates can react with oxygen, even while the machine stops. Therefore, the radical intermediates should be deactivated as soon as they were generated.

The radical scavengers can deactivate the active intermediates, thereby breaking the chain reaction. Phenol derivatives are commonly used for this role [74-76]. Even with these reagents, a small portion of radical species can react with oxygen and yield peroxide. In other words, the function of the radical scavengers is not always perfect in preventing the autoxidation reaction. Organic peroxides possess strong oxidative attack to other organic molecules and metallic materials. Aromatic amines [77,78], alkyl sulfides [79], and ZnDTP [80] are frequently employed to decompose peroxy radicals or peroxides. Peroxide decomposers are post-active AO that deactivate the active intermediates after the reaction with oxygen. On the other hand, radical scavengers and metal deactivators are proactive AO that suppress the reaction prior to the oxidation step.

It is empirically known that certain metal catalyzes the autoxidation of hydrocarbons. The wear process produces nascent surfaces that display catalytic activities. The surface reactions become significant when tiny wear particles are dispersed in the lubricant, because they have large surface area. Metal deactivators can interact with metal surfaces and block the active sites on the surfaces. From the mechanistic viewpoint, metal deactivators work at a solid-liquid interface and the others work in the liquid phase. 


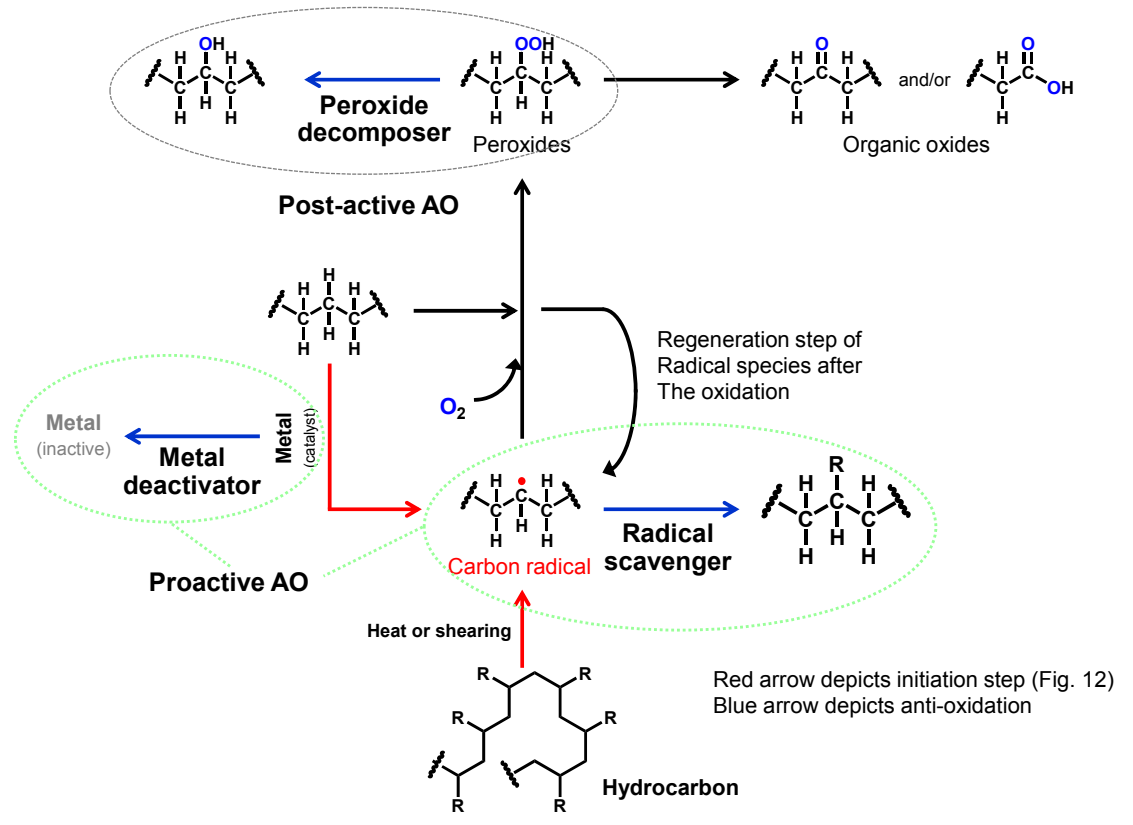

Figure 13. Oxidation of lubricants and working mechanism of AO (in ellipse).

Different types of AOs are frequently used together in a lubricant [81]. Since tribological processes usually induce different causes of autoxidation, different $\mathrm{AO}$ complement each other to prevent the ageing processes happening simultaneously.

\subsubsection{Detergent}

Several tribo-improvers contain nitrogen, phosphorus, or sulfur atoms in the molecule. Oxidation of these compounds results in mineral acids and their derivatives. These acidic compounds can attack tribo-materials and/or further promote the ageing of the lubricant. Detergents neutralize or deactivate these aggressive chemicals (Figure 14) [82]. Fine particles of calcium carbonate could be dispersed in hydrocarbons with the aid of surfactant. This releases the alkali salt upon formation of acidic compounds. Because of the structural features, they are called "over-based type detergent". The base number and mean particle size are the measure of the quality for these additives.

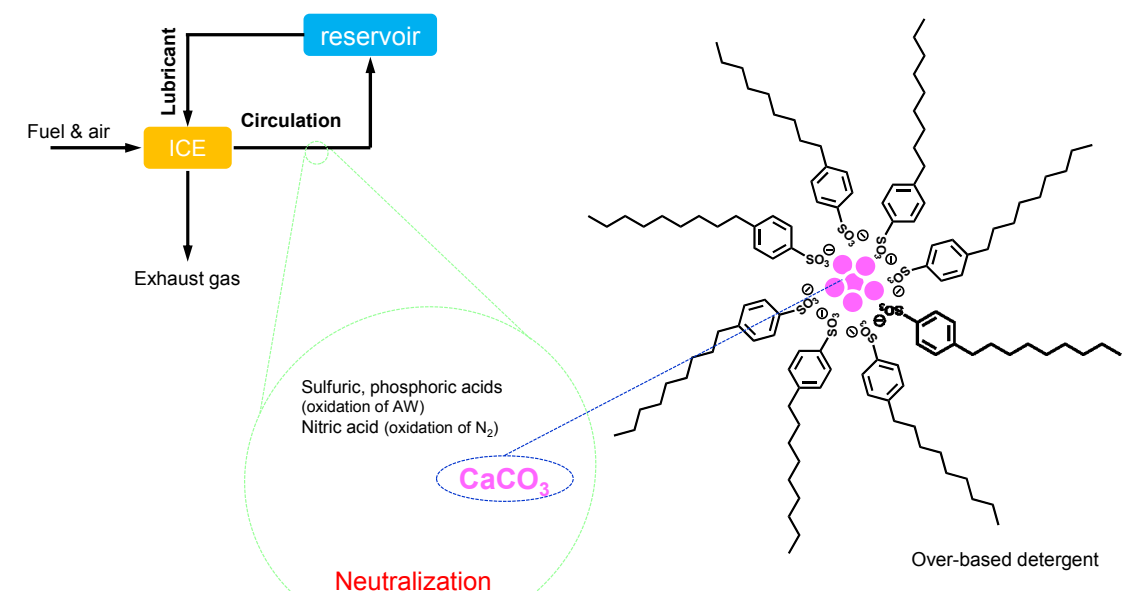

Figure 14. Working mechanism of detergent (example diesel engine oils). 


\subsubsection{Dispersant}

An internal combustion engine often yields soot. When it disperses in the lubricant, abrasive wear, damaged seals, or depleted tribo-improvers may occur [83]. When the ageing proceeds further, organic oxides are converted to polymers. This yields insoluble matters or deposits. In contrast to wear particles of metallic materials, these oligomers and polymers are gel-like and are not easily filtered off. A dispersant molecule can interact with these organic contaminants and disperse in the liquid phase (Figure 15) [84]. The primary function of dispersants is surfactant, which is analogous to detergent. However, the dispersant molecule has more polar groups in order to capture organic contaminants sufficiently and to keep it dispersed in the liquid phase.

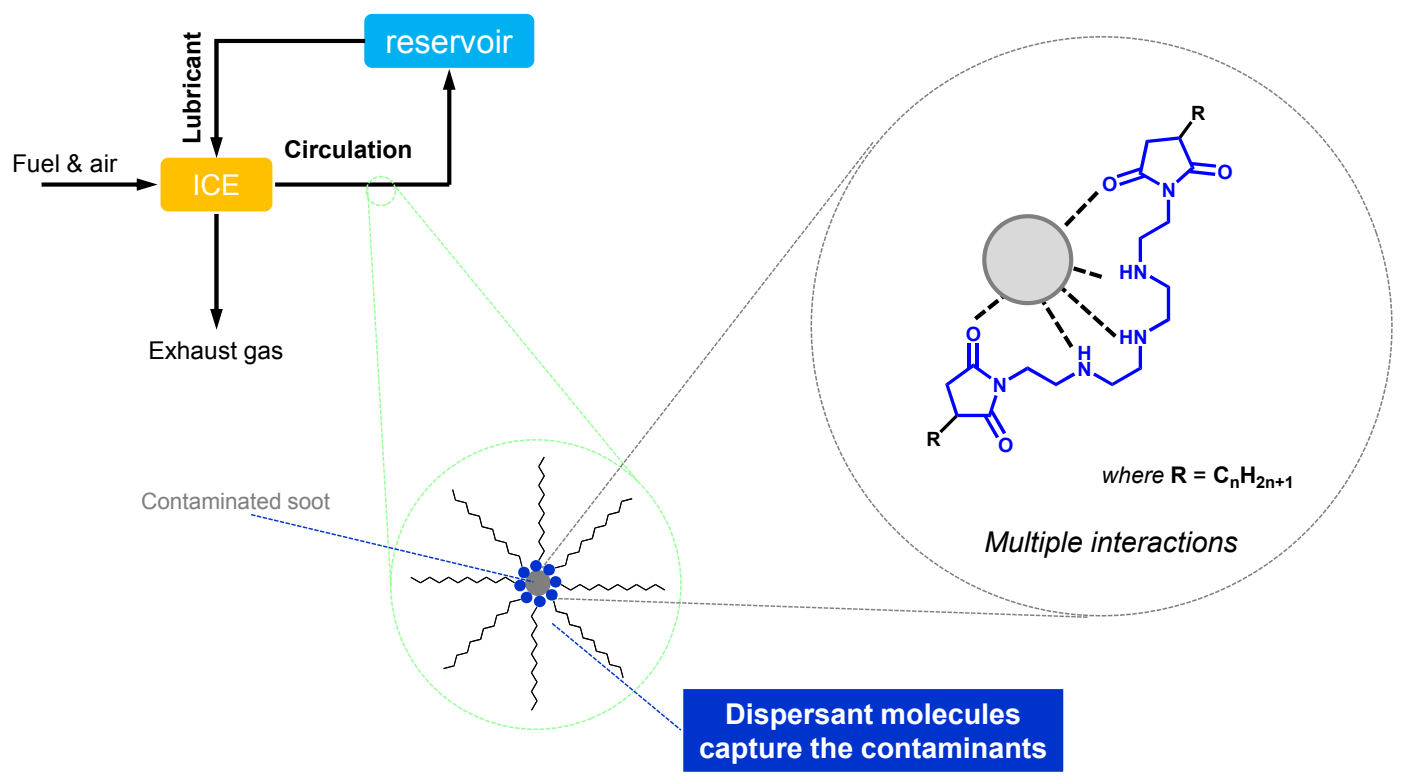

Figure 15. Working mechanism of dispersant (example diesel engine oils).

\subsubsection{Corrosion Inhibitor, Rust Preventive}

Corrosion and rust are defined as an oxidative degradation of metallic materials through electrochemical processes. The Galvanic corrosion process is the representative corrosion process in lubrication systems. The mechanism in brief is as follows. Metal surfaces are usually covered with an oxide layer. The tribological process wears the oxide off and exposes metal surfaces. The electrical potential between metal and metal oxide is different. When a conductive liquid exists between the surfaces, Galvanic cell is formed. The metal atom liberates electrons and forms metal ion by increasing the oxidation number (this process is the oxidation of metal). The electron liberated from the metal reduces an oxygen molecule in the system (Figure 16). Base fluids (hydrocarbons) are insulators but some additives can increase the electrical conductivity of the lubricant. Another cause of corrosion is the ionization of metal by acidic compounds that occur without oxygen. Both corrosion mechanisms can take place under static conditions while the machine is pausing. Corrosion inhibitors provide a protecting layer on metal surfaces [85]. The protection layers could be either organic (adsorption type, providing organic layers) or inorganic (reaction type, providing passive layers), which are analogous to FMs (see Section 2.1.1). In contrast to FMs, those protecting films from corrosion do not need mechanical robustness. 


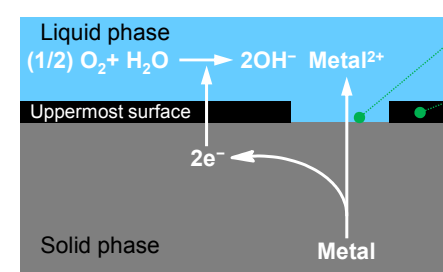

Galvanic corrosion
Worn surface

Non-rubbed surface

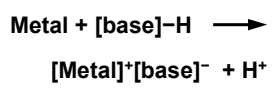

Ionization with acids

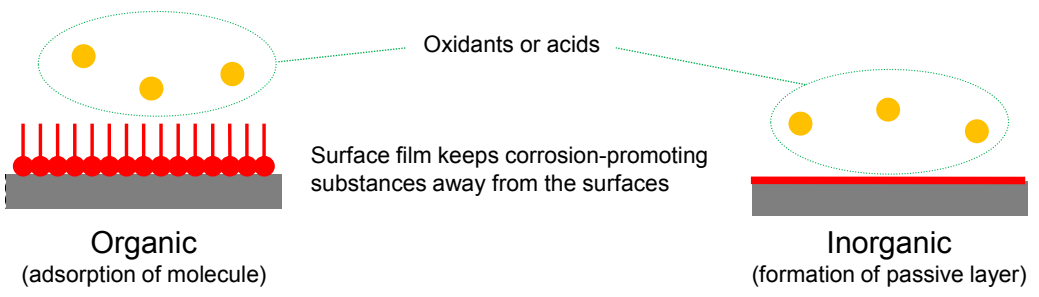

Figure 16. Corrosion of metal and the working mechanism of corrosion inhibitor.

\subsubsection{Anti-Foam Agent (Defoamer)}

Lubricants can intake air during work and circulation. This develops an air bubble in the lubricants, and results in drop of viscosity [86]. In addition to the physical changes, the autoxidation of base oils is facilitated because the probability of oil-oxygen contact is increased through an extended area of the gas-liquid interface. In this regard, non-visible tiny bubbles are problematic. Anti-form agents are composed of surfactant-like molecules that can interact between gas-liquid interfaces. The function is to merge tiny bubbles into a large one, thereby helping to release the molecule from the liquid phase (Figure 17) [87].

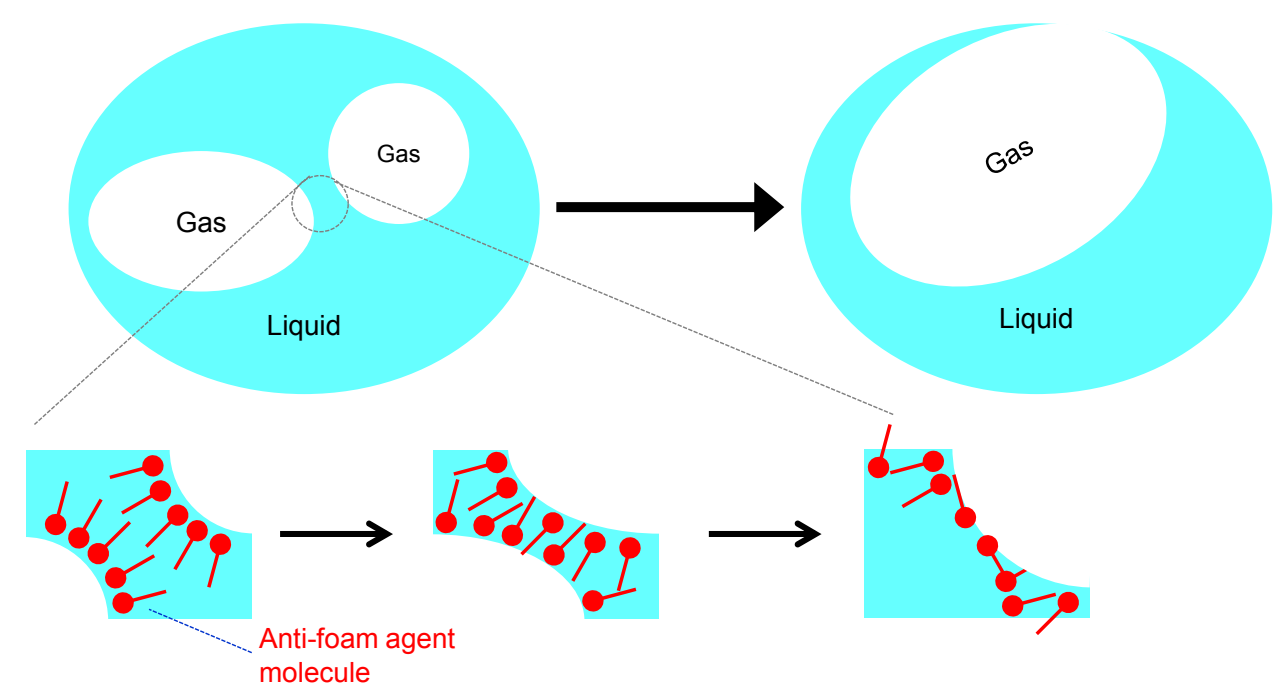

The surfactant promotes the coalescing of tiny bubbles into large ones. As a result, large bubbles can rise to the surface and pop.

Figure 17. Working mechanism of anti-foam agent (defoamer).

\subsubsection{Demulsifiers (Emulsion Breaking Agents)}

Water is a ubiquitous contaminant for almost all lubricants. It causes many problems in lubrication systems including viscosity drop, corrosion of metallic materials, hydrolysis of additives, etc. Therefore, lubrication systems are better if they have water release systems from the lubricant. It is reasonable to drain contaminated water from the lubricant (density less than 1) while the lubricant is settling in 
a reservoir. As discussed supra, and summarized in Table 2, many additives work at the interface. These additives have surfactant nature more or less. Therefore, water can diffuse in the lubricant in the form of water-in-oil emulsion with the aid of those additives. Demulsifiers break water-in-oil emulsion by deactivating the surfactant type additives as illustrated in Figure 18.

The role of anti-foam agents and demulsifiers is to release contamination, gas or liquid respectively, from the lubricants. However, they work in the opposite way; anti-foam agents activate the gas-liquid interface, while demulsifiers deactivate the water-oil interface.

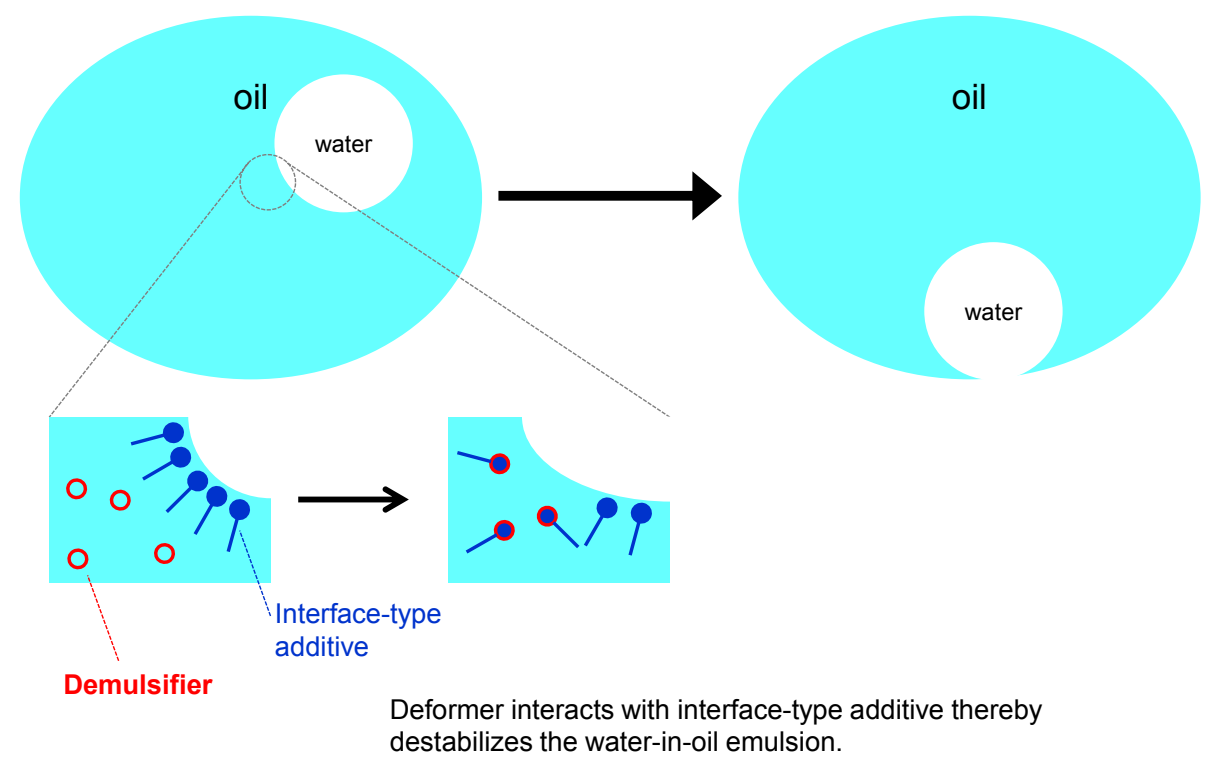

Figure 18. Working mechanism of Demulsifier.

\subsection{Multi-Functional Additives}

Some additives have inter-category functions, i.e., display more than two roles out of three (tribo-improving, rheo-improving, and maintaining, see graphic abstract and Table 2) functions. ZnDTPs are well-known and the most widely used additives in lubricants that act as FM, AW, AO, and corrosion inhibitors, depending on the structure of the alkyl group [88,89]. This means that ZnDTPs take the role as both tribo-improvers and maintainers. Other examples in practices are: over-based calcium sulfonates as detergent and AW [90], and functionalized poly(methacrylate) as VM and $\mathrm{AW}$ [91].

\subsection{Auxiliaries}

Not the all but some specific lubricant may include certain substances that are not categorized as the major groups.

\subsubsection{Antibiotics}

High water content fluids could be used for some hydraulic fluids or metal working fluids. These fluids occasionally experience biological decay during storage. Antibiotics can prevent ageing.

\subsubsection{Conductivity Improver}

These additives are not very common but required for certain applications. The conductivity is one of the bulk liquid properties. Hydrocarbons, most commonly used as base oils, possess relatively poor thermal conductivity. Although this property is of importance for the cooling effect, other advantages with hydrocarbons are usually considered. Dispersion of inorganic particles may increase the conductivity [92]. 


\subsubsection{Colorings}

This ingredient is not necessary for lubrication performances but is sometimes beneficial for lubricant maintenance. Specific coloring can prevent mistakes by mixing wrong lubricant when replacement or replenishment is taken. In some applications, leakage could be found easily by coloring of the liquid.

\section{Evaluation of Tribo-Improvers}

Even if a researcher develops an excellent additive, it would be never commercialized directly. The R\&D processes of lubricants has been undertaken step-by-step as briefly shown in Table 3. Among three additive categories, rheo-improvers and maintainers are analogous substances for other industrial materials. Development and primary evaluation of these additives could follow the procedure for those materials. On the other hand, tribo-improvers are unique to lubricants. This section focuses on tribo-improvers.

Table 3. Research phase and tribo-testing.

\begin{tabular}{|c|c|c|c|c|c|c|c|}
\hline R\&D Phase & Focus & $\begin{array}{c}\text { Test } \\
\text { Category }\end{array}$ & $\begin{array}{c}\text { Sample } \\
\text { Lubricant }\end{array}$ & Test Equipment & Outcome & $\begin{array}{c}\text { Test } \\
\text { Periods }\end{array}$ & $\begin{array}{c}\text { Cost of } \\
\text { Evaluation }\end{array}$ \\
\hline $\begin{array}{c}\text { Incubate-break } \\
\text { through }\end{array}$ & Possibility & \multirow{2}{*}{$\begin{array}{l}\text { Laboratory } \\
\text { test }\end{array}$} & $\begin{array}{c}\text { Specific } \\
\text { components }\end{array}$ & Universal tribo-tester & $\begin{array}{l}\text { The role } \\
\text { component }\end{array}$ & \multirow{4}{*}{$\begin{array}{c}\frac{+}{0} \\
\frac{0}{\omega}\end{array}$} & \multirow{4}{*}{ 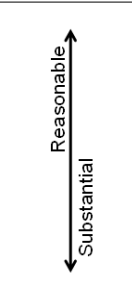 } \\
\hline Improve & Applicability & & Prototype & $\begin{array}{l}\text { Tribo-tester according to } \\
\text { industrial standards }\end{array}$ & $\begin{array}{c}\text { Technical } \\
\text { benefit }\end{array}$ & & \\
\hline Optimize & Feasibility & Bench test & \multirow{2}{*}{$\begin{array}{c}\text { Full } \\
\text { formulated }\end{array}$} & Machine component & $\begin{array}{c}\text { Engineering } \\
\text { benefit }\end{array}$ & & \\
\hline Verity \& tune & Productivity & Field test & & Real machine & $\begin{array}{c}\text { Industrial } \\
\text { benefit }\end{array}$ & & \\
\hline
\end{tabular}

Because of variety of machine elements-their configurations, operating conditions, and environment-lubricants should be formulated for each machine element. The performances of these lubricants are evaluated using the practical machine under real conditions and environments. This so-called field test is a time- and cost-intense procedure and is usually employed just before the introduction of the product into the market. Prior to the field test, lubricants have to pass the laboratory tests that are classified as "bench tests". This tests lubricants using the real machine elements (part of whole machine) under simulated operating conditions and environments in the laboratory. These tests should focus on optimizing, verifying or tuning of the machine. Therefore, fully formulated lubricants are employed for evaluation. Since these phases test long-term durability and safety of the machine, requirements for lubricants are close to practical qualities. The first two phases in R\&D are carried out using laboratory equipment in a time- and cost-effective manner. Standard tests regulated by the authorized organizations such as ASTM are usually employed in these early research stages. Each standard test clearly defines the equipment and the procedure and mainly focuses on the quality control of industrial materials. Another advantage of the standard tests is that equipment and related parts including consumables are commercially available in most cases. Therefore, these tests are also employed for evaluation of prototype lubricants in an "improving" phase.

It should be noted that the standard tests define the minimum requirements as industrial products for individual applications. These procedures are supposed to evaluate fully formulated lubricants. Therefore, these procedures may involve over-loaded conditions for lubricant additives under an incubate-breakthrough phase.

Contrary to well-established methods and apparatuses for chemical analysis such as spectroscopies, there is no consensus as to a universal tribo-testing procedure for tribo-improvers in laboratories. Simple tribo-testers (not a part of machine element) are beneficial in an incubating phase. Factors to be considered in conducting a tribo-test of lubricants are described below. 
- Configuration of tribo-contact: a point contact generates high contact stress while line and square contact generate low contact stress. A careful setup of the test specimen is needed to ensure the alignment of contact. This influences the repeatability of the test considerably. Ball-on-flat type point contact provides a comparatively easy setup.

- Type of relative motion: Reciprocation sliding, unidirectional sliding, rolling, and a combination of rolling and sliding are most commonly employed.

- Tribo-materials: Various materials are used in different machine elements. Simple tribo-tests are beneficial for evaluating the compatibility of tribo-improvers with specific materials in machine elements. Surface roughness of the materials should be considered.

- Operating conditions: Load, velocity, and temperature of lubricant should be controlled in a proper way.

- Testing environment: Should be kept away from contaminants as much as possible to obtain the results with good repeatability. Humidity of room air can influence the tribological properties.

In summary, there are countless test conditions that combine the above factors. Under these circumstances, simple comparison of the results, i.e., friction coefficient or wear rate, between different groups does not always make sense. Performances of tribo-improvers should be evaluated by comparing the base fluid (without the tribo-improver, see Figure 4).

\section{Discussion as Multi-Component Systems}

\subsection{Formulation of a Lubricant}

Based on the understandings of each component for lubricants, this section discusses the combination of additives to achieve lubrication performances. Manufacturing a lubricant, so-called "formulation", requires high-level engineering knowledge; what base oil(s) and additives should be chosen? What is the concentration of each component?-The contents in lubricants are in a black box in most cases. It seems an implicit agreement that everybody can produce a fake-lubricant if the detailed contents in a lubricant were disclosed. That might indeed be possible but this is not a good reason to attempt this. Here is a simple question; can you serve a high-quality dinner by merely following the recipe of a three-star Michelin restaurant? Note that lubricants cannot be formulated by simple mixing of base fluids and additives. The reason for non-disclosure of lubricant contents might be an economical matter. Everybody can calculate the total cost of the raw materials in a lubricant and then might feel that the product price is too expensive, without considering the cost for the quality. The cost discussion is not in the scope of this review and the following sections highlight the science of additive technology.

\subsection{The Quality of Each Ingredient}

The total performances of formulated lubricant are often influenced by the quality of each component. In chemistry, quality of substance is nearly equal to the purity. In this regard, it is a tacit knowledge that even a small amount of impurities sometimes play a decisive role, especially in tribological performances. This phenomenon could be understood by a competitive interaction of impurities against the tribo-improvers with tribological surfaces. Actually, many lubricants are made of industrial grade chemicals that are usually less pure compared to reagent grade. Laboratory tribo-tests are a reasonable way to check the quality of the ingredients of lubricants.

\subsection{The Interaction of Ingredients}

Because of the nature of multiple components in a lubricant, an interaction between each component (either as additive or as base oil) occurs inherently. The interaction can result in a positive, negative, or neutral effect on lubrication performances including lifetime of usage. A positive effect is a synergistic effect; improved performance by combination compared to one component alone. 
Creating new function(s) that were not provided by each ingredient is also within this category. The opposite case is an antagonistic effect. In the neutral case, each ingredient works independently, even though they interact with each other in a molecular level.

Antagonism of ingredients is frequently observed in preparation and evaluation of prototype lubricants. A simple example is shown in Figure 19; each tribo-improver A or B prevented wear by a laboratory test, while mixing them both displayed higher wear than the base oil alone. Antagonism should be notified if $A+B$ displays much more wear than B (not base oil), in this example. A reaction of A with B was suggested in this case [93]. Depletion of tribo-improvers by dispersants [94,95] is categorized as antagonism in a broad sense.

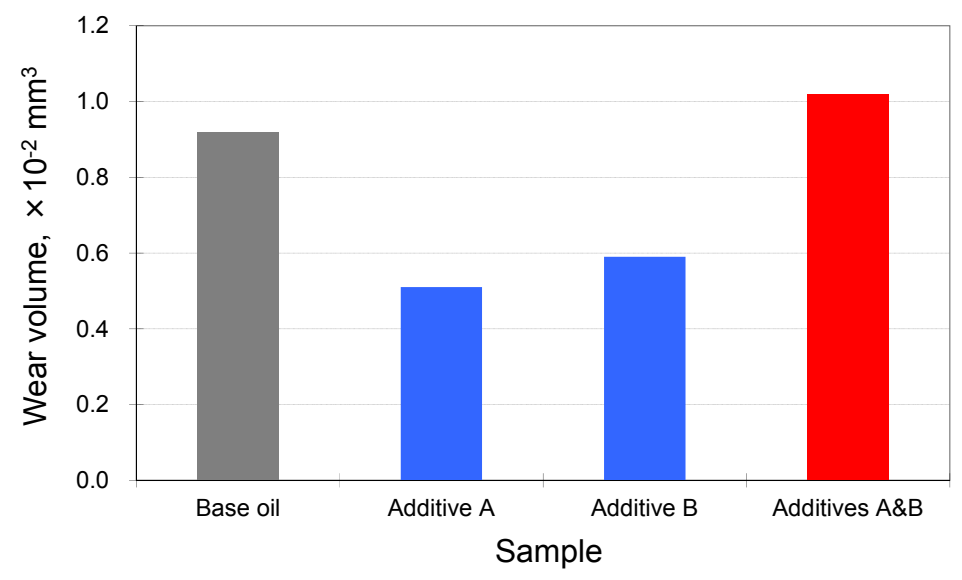

Figure 19. Antagonism of two additives.

The term "synergism" seems to be often used in the literature, aiming to show the result is positive. Here we introduce a clear definition for lubricant chemistry. In molecular science, the molarity of a substance (unit as $\mathrm{mol} \cdot \mathrm{kg}^{-1}$ ) is important. Therefore, the lubrication performance should be compared using samples with the same molarity of the additive. In short, "synergism" should be stated as an improvement by combination, if the total molarity of the combined ingredients is exactly same as the single component. Improvement by simply mixing could be the effect of the total amount of ingredients (better performances by higher concentration of ingredients), as concisely illustrated in Figure 20. In this regard, mass concentration is the most common unit in lubrication engineering. Therefore, it is better to reconsider the published results.

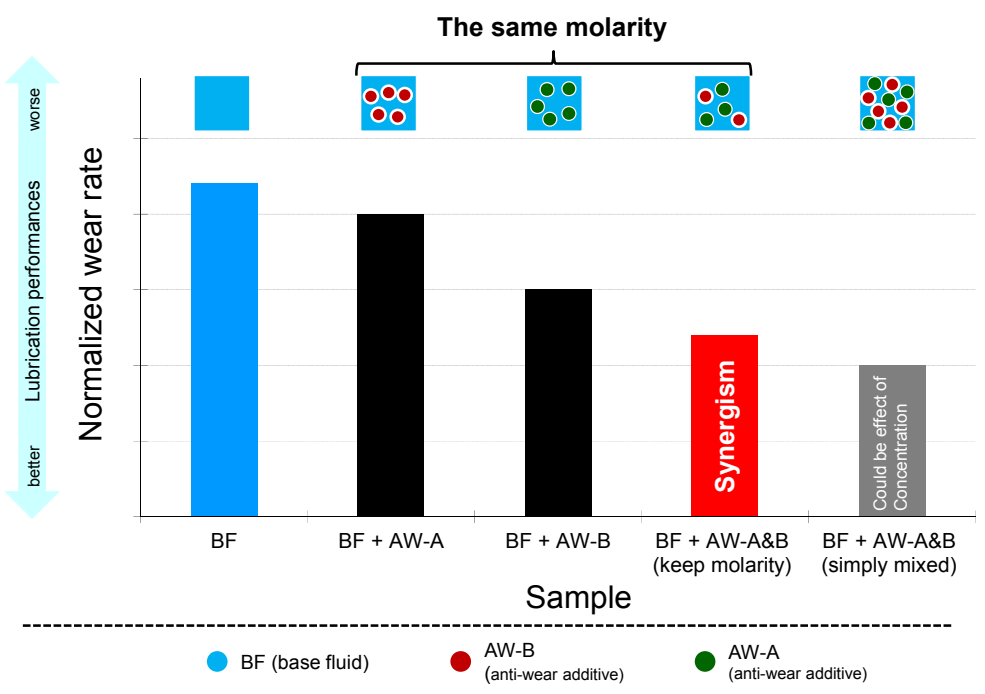

Figure 20. Synergism model (example of AW). 
It is uncertain in terms of engineering knowledge whether a combination of tribo-improvers, e.g., FM and AW, can provide better lubrication performances. This could be classified into 3 phenomena, as illustrated in Figure 21.

- Synergy is defined as the combination improves BOTH friction and wear compared to each ingredient alone.

- Enhanced performance of one while scarifying the other seems to have an improved effect but should not be defined as synergism.

- $\quad$ Antagonism is any other phenomena except the above two.

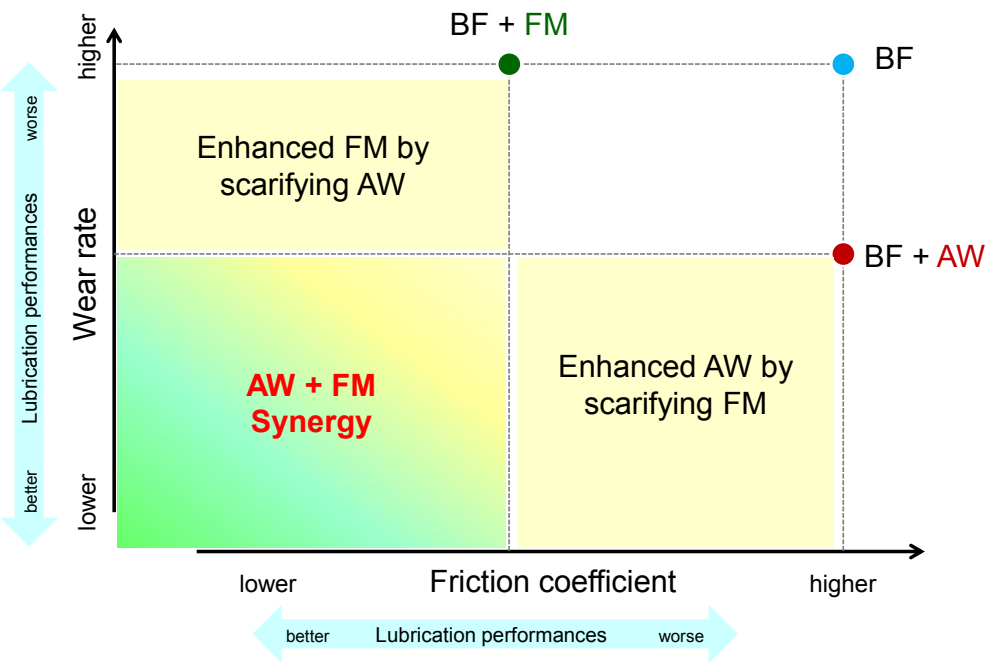

Figure 21. Synergy chart (example of FM and AW).

As is common knowledge, different AOs (radical scavengers, peroxide decomposers, and metal deactivators) are dissolved in a lubricant [83]. From a mechanistic viewpoint, these AOs are on standby for different causes of autoxidation (Figures 12 and 13). Therefore, this is understood by the complemented effect.

The interaction of different additives highly concerns the formulation process of a lubricant. It occasionally happens that the lubrication performances can differ by the order of a mixing process. Although this involves high-level engineering knowledge in formulating a lubricant, the phenomena could be explained by the interaction of additives, as illustrated in Figure 22. As discussed supra (Table 2), a lubricant contains different surfactant-type molecules that work at interfaces. Because of the "interface activating nature", these molecules aggregate themselves or incorporate other additives.

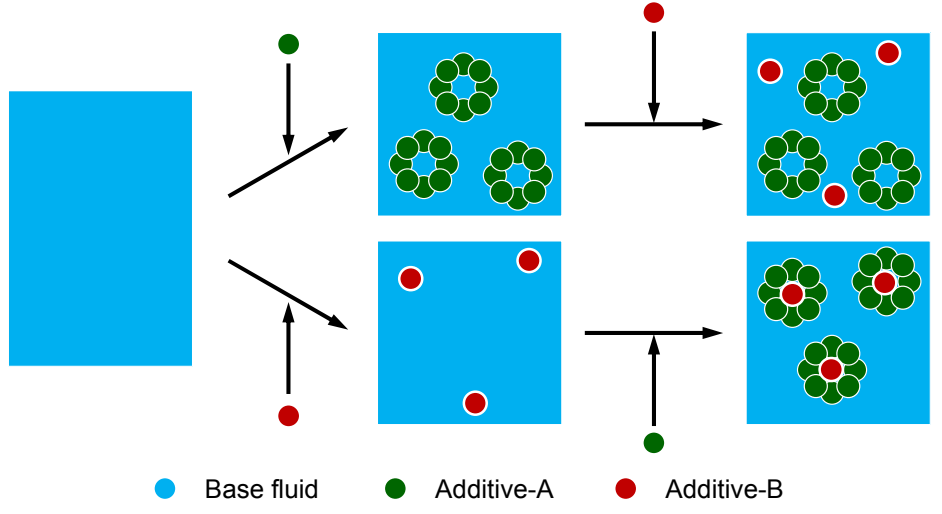

Figure 22. Possible variation of performance by formulation process. 


\subsection{Additive Technology for Synthetic Fluids}

Historically, additive technology in lubrication engineering arose in the early period of the petroleum age. Mass production of mineral oils, together with naphtha and fuels from crude oils, provided considerable benefits in the market. Liquids with proper viscosity for lubrication had become available at reasonable costs. However, neat mineral oils (without additives) usually display poor lubrication performances, especially in reducing friction and wear, in comparison with natural triglycerides (plant oils and animal tallow that had been the resources of lubricants since the ancient days until the petroleum age). After the advantage of additives had been recognized, the demands of additive technology sprang up. It was proved in many applications that additive technology enables machine operation with enhanced reliability, improved energy efficiency, and prolonged lifetime, etc. It should be emphasized that the improvements in lubricant performance motivated an advanced design of machine elements. This further promoted the improvement of lubricant performances. Today and in the future, additive technology is and will be indispensable in lubrication systems.

The majority of base oils in the lubricant industry had been and is still Group I oils. Therefore, additive technology has been focused on improvement of Group I oils. Demands of quality lubricants from users are promoting the shift of base fluids to Group II or III from Group I. Then the problem with the solubility of additives in these base fluids arose. The same problem happens with Group IV. Although Groups I-IV are composed of hydrocarbons, Group I oils contain a considerable amount of cycloalkanes (naphthenic) and aromatics that are responsible for good solvency. Solvency-improving fluids are usually blended in these poor solvents (Group II-IV oils).

Solvency could be understood by the affinity of a solute to the solvent. If a compound does not dissolve in the base oil, formulation of a liquid lubricant is impossible. On the other hand, high solubility of a solute in the solvent is not desirable. A proper solubility, especially for tribo-improvers, is required in formulating a lubricant. Figure 23 depicts a model of solubility-adsorptivity relation on lubrication performance, in consideration with an adsorption isotherm. From the working mechanism of tribo-improvers (Figures 5-7), it is possible to ascertain that they start by adsorption on rubbing surfaces. Assume that there is a threshold of surface coverage at $C$ by which adsorption enables the tribo-improver to work. When additive- $\mathrm{A}$ and additive- $\mathrm{B}$ were compared at the same concentration of XA, the former displays expected performances while the later displays none, due to insufficient amount of molecules on the surface. A higher concentration of $X B$ is needed for additive-B to perform. However, using an additive at high concentration rises the risk of disadvantages by the interaction with other additives. Therefore, additives should have proper solvency to the base fluids. The solubility in the base fluid is the second key factor in choosing additives after the primary lubrication function.

Esters and polyethers belong to Group V oils and are receiving growing demands as fast biodegradable fluids [96]. Contrary to Groups I-IV oils, they contain oxygen atoms in their molecule. Here we discuss the difference between hydrocarbon and oxygen-containing molecules (esters and polyethers) in terms of polarity. Polarity is a result of unequaled sharing of the bonding electron pair, due to the differences in electron negativity of the atoms that form the covalent bond. The electron negativity of hydrogen, carbon, and oxygen are 2.2, 2.5, and 3.5, respectively. Hydrocarbons are being constructed with the combination of a carbon-carbon bond and carbon-hydrogen bonds. Since the differences in the electron negativity of the atoms of these bonds are low ( 0 for $\mathrm{C}-\mathrm{C}$ bond, 0.3 for $\mathrm{C}-\mathrm{H}$ bond), hydrocarbons display "non-polar" properties. On the other hand, esters and polyethers have carbon-oxygen bonds (the difference in the electron negativity is 1.0), together with $C-C$ and $\mathrm{C}-\mathrm{H}$ bonds. Some substances involve hydrogen-oxygen bond(s) in which the difference in the electron negativity is 1.3. These oxygen involving bonds make the molecule polar. Furthermore, the chemical structure should be considered for the polarity of the molecule, which is omitted here for simplicity.

Generally, polar liquids are good solvents and hence esters and polyethers can dissolve common additives for Groups I oils up to high concentrations. This is an analogous problem to that of additive-B in Figure 23. By and large, polar molecules display an affinity to steel surfaces. This induces adsorption of polar fluids on tribo-surfaces. Here, competitive adsorption of a base fluid against a tribo-improver 
occurs. When the base fluid molecules adsorb on the surface and block the surface from being interacted with the additive, additive effects will not result.

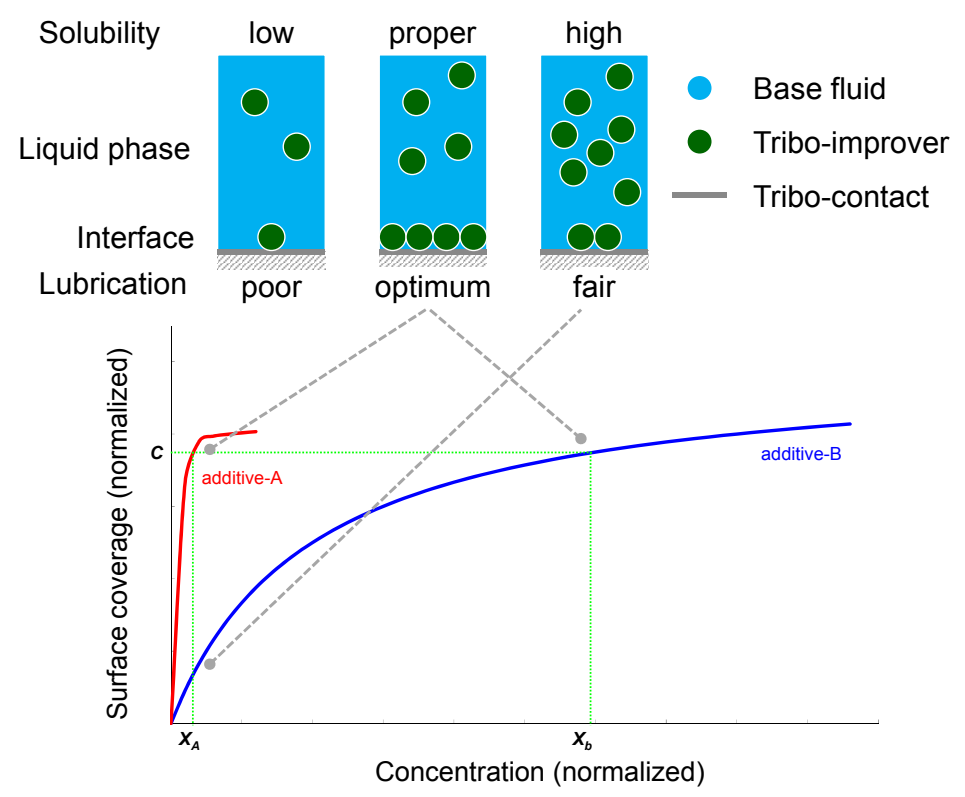

Figure 23. Solubility of a tribo-improver and lubrication performance.

It was anticipated that increased polarity in an additive molecule can enhance the adsorption activity from solution in a polar solvent. Examples have enhanced polarity by chemical modification of phosphates such as AW for polar synthetic fluids, as shown in Figure 24 [97]. This prevented wear at reasonable concentrations, while conventional AW needs a much higher concentration to work in polyether. Conventional AWs usually work in hydrocarbon oils at a concentration of $10 \mathrm{mmol} \cdot \mathrm{kg}^{-1}$ (310 ppm of phosphorus content) or below.

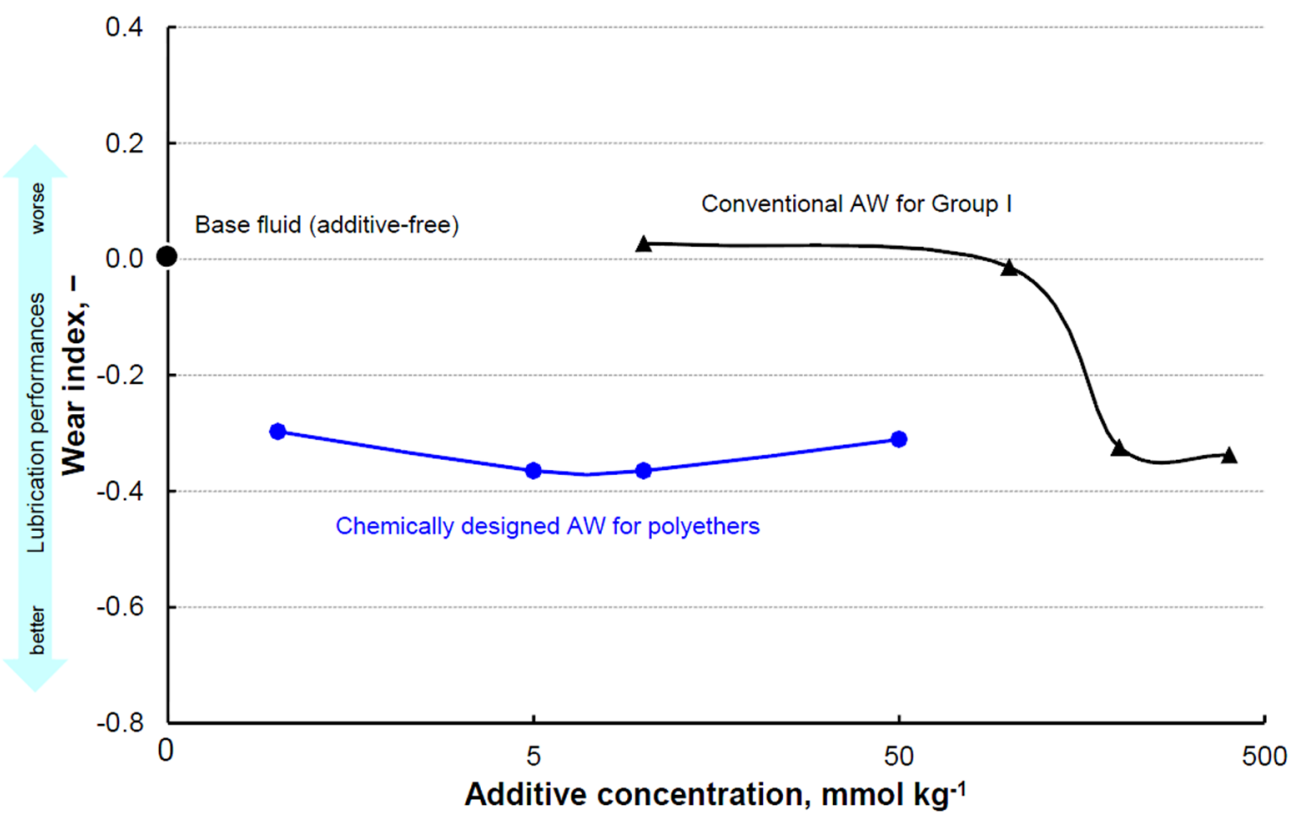

Revised from figure 3 in ref 97.

Figure 24. Performances of different AW in synthetic fluid (polyether). 
The relation between chemical structure and physical properties for synthetic esters has been reviewed in [98]. Non-polarity index was introduced therein as an engineering parameter. The performance of model AW for polar synthetic fluids were evaluated and analyzed regarding polarity index as minor revision of non-polarity index. As shown in Figure 25, performance of the anti-wear properties decreases as the polarity of the base fluid increases [99].

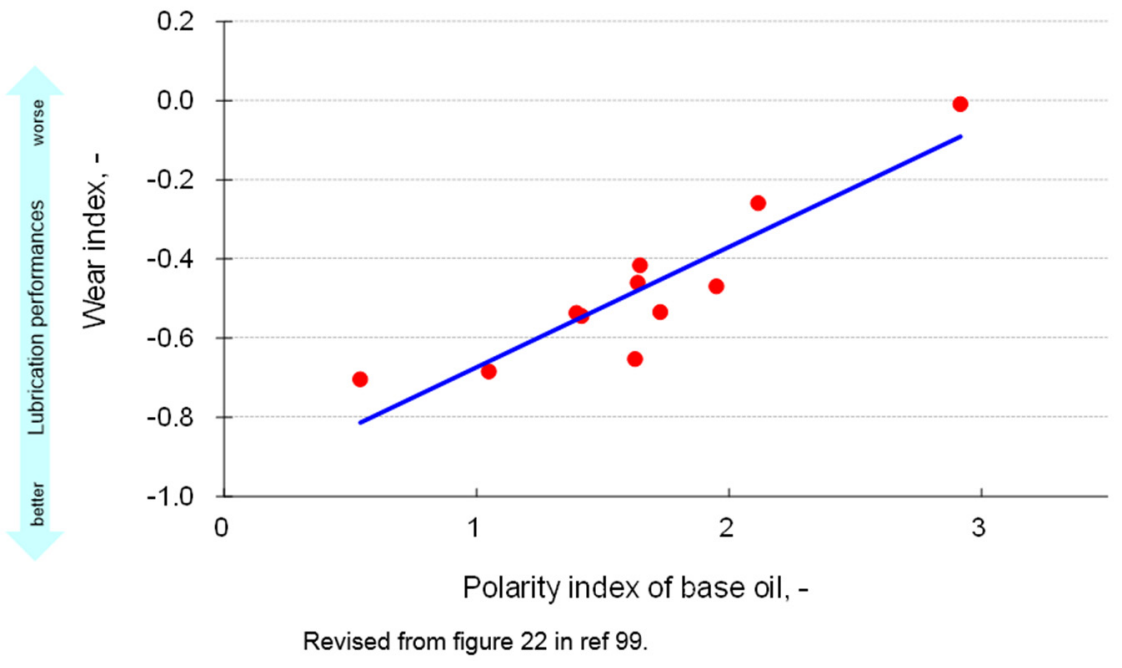

Figure 25. AW performances for different synthetic fluids (ester).

\subsection{Tribo-Improvers for Non-Ferrous Materials}

Most tribo-improvers are developed for lubrication of steels. When it comes to other materials, affinity of each additive to the material is different from that of steel. Therefore, the kinetics in the adsorption step (Figure 6) would be influenced. The processes of tribo-chemical reaction and the property of the tribo-film could be different from those with steel. Various materials including coatings are being developed for tribological purposes. Molecular structure of tribo-improvers should be tuned for the materials.

\subsection{Additive Technology for Environmentally Acceptable Lubricants}

Because of the concern for reducing the impacts on the global environment, demands of EAL (environmentally acceptable/adapted lubricants) are increasing. The biodegradability is one of the key factors in choosing base fluids for EAL [96]. Synthetic esters, natural triglycerides, polyethers, and certain synthetic hydrocarbons are candidates of the base fluids in EALs. Significant investigation in additive technology is needed, especially in the solubility of additives, as discussed in the previous section. Besides the problem of solubility and adsorption for synthetic fluids, toxicity and bioaccumulation tendency of additives are also a concern. Although the contents are minor portions, it has been pointed out that certain ingredients in industrial products should be or might be better if replaced by safer substances [100]. "Greener" candidates have been proposed for 10 specific cases therein. Among them, antimicrobials, small amines, boron alternatives, corrosion inhibitors, alkanolamides, and surfactants relate to lubricant chemistry.

\section{Summary}

Even though they constitute the minor contents in a lubricant, additives are responsible for various functions in lubricating a machine element. The functions include not only tribological properties but also the material properties and maintenance. Many machines are in use under various lubrication conditions. Therefore, the required functions of each lubricant are different. This leads to a vast variety of commercial lubricants. Publications in lubrication engineering and lubricant chemistries 
are mostly focused on individual engineering phenomena. Unfortunately, scientific viewpoints have thus far underestimated such lubricant technology. This review helps the readers to survey the additive technology for lubricants. The main role and functions of individual additive category were introduced with an emphasis on molecular science. Similarity and dissimilarity in functions and molecular structure were discussed by comparing different additive categories.

The continuous developments in machine elements require advanced lubricants more and more. The improvement in efficiency, reliability, and lifetime together with environmental acceptability are core concerns. These technological requirements should be solved by additive technology. The availability of synthetic fluids and new tribo-materials open the opportunities for designing advanced machine elements. This requires a much bigger lineup of task-specific lubricants. This would constitute a paradigm shift from empirical knowhow to a smart technology in research and development of advanced lubricants. Here we wish to propose the algorithm of molecular science in the R\&D of lubricant chemistry. Lubricant chemistry is an ever-improving science.

Acknowledgments: A part of this work is supported by Maritime Technologies II Research Programme in the framework of the ERA-NET MARTEC II project CA 266111 ArTEco 'Arctic Thruster Ecosystem' and "Austrian COMET-Program" in the frame of K2 XTribology (project No. 849109) within the "Excellence Centre of Tribology" $\left(\mathrm{AC}^{2} \mathrm{~T}\right.$ research $\left.\mathrm{GmbH}\right)$.

Conflicts of Interest: The author declares no conflict of interest.

\section{Nomenclature}

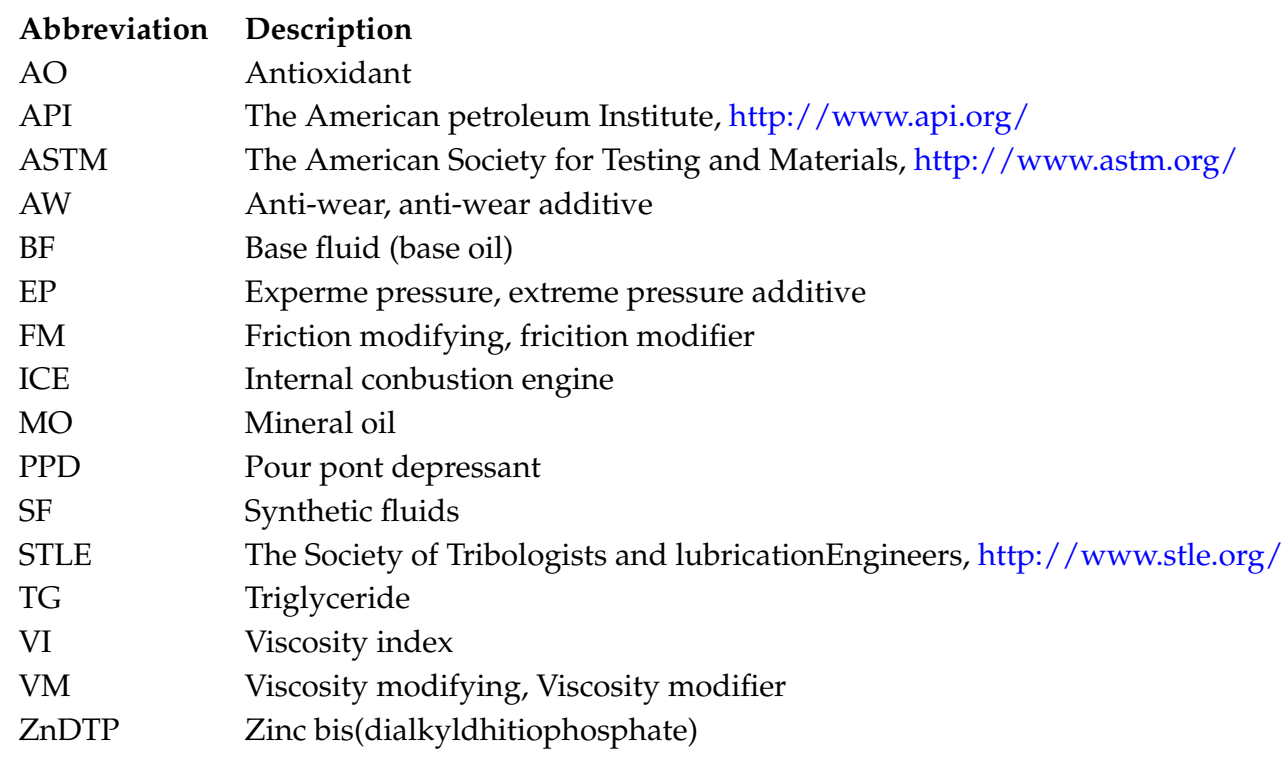

\section{References}

1. Stevenson, A. "Tribology" in Oxford Dictionary of English, 3rd ed.; Oxford University Press: Oxford, UK, 2015.

2. Jost, H.P. "Tribology or Lubrication" in Lubrication (Tribology) Education and Research; Department of Education and Science, Her Majesty's Stationery Office London: London, UK, 1966.

3. Dowson, D. "The Early Civilizations" (Chapter 4) in History of Tribology, 2nd ed.; Professional Engineering Publishing: London, UK, 1998.

4. Wang, Y.; Wang, Q.J. "Stribeck Curves" in Encyclopedia of Tribology; Wang, Q.J., Chung, Y.W., Eds.; Springer: New York, NY, USA, 2013; Volume 5.

5. Zhu, D.; Wang, Q.J. "EHL History (Elastohydrodynamic Lubrication)" in Encyclopedia of Tribology; Wang, Q.J., Chung, Y.W., Eds.; Springer: New York, NY, USA, 2013; Volume 2.

6. Rennie, R. "Viscosity" in Oxford Dictionary of Chemistry, 7th ed.; Oxford University Press: Oxford, UK, 2016.

7. Kaupp, G. Mechanochemistry: The varied applications of mechanical bond-breaking. CrystEngComm 2009, 11, 388-403. [CrossRef] 
8. Takacs, L. The historical development of mechanochemistry. Chem. Soc. Rev. 2013, 42, 7649-7659. [CrossRef] [PubMed]

9. Cravotto, G.; Gaudino, E.C.; Cintas, P. On the mechanochemical activation by ultrasound. Chem. Soc. Rev. 2013, 42, 7521-7534. [CrossRef] [PubMed]

10. Šepelák, V.; Düvel, A.; Wilkening, M.; Becker, K.-D.; Heitjans, P. Mechanochemical reactions and syntheses of oxides. Chem. Soc. Rev. 2013, 42, 7507-7520. [CrossRef] [PubMed]

11. Ribas-Arino, J.; Marx, D. Covalent Mechanochemistry: Theoretical Concepts and Computational Tools with Applications to Molecular Nanomechanics. Chem. Rev. 2012, 112, 5412-5487. [CrossRef] [PubMed]

12. Li, J.; Nagamani, C.; Moore, J.S. Polymer Mechanochemistry: From Destructive to Productive. Acc. Chem. Res. 2015, 48, 2181-2190. [CrossRef] [PubMed]

13. Todres, Z.V. Organic Mechanochemistry and Its Practical Applications; CRC Press Inc.: Boca Raton, FL, USA, 2006.

14. James, S.L.; Adams, C.J.; Bolm, C.; Braga, D.; Collier, P.; Friščić, T.; Grepioni, F.; Harris, K.D.M.; Hyett, G.; Jones, W.; et al. Mechanochemistry: Opportunities for new and cleaner synthesis. Chem. Soc. Rev. 2012, 41, 413-447. [CrossRef] [PubMed]

15. Birke, V.; Mattik, J.; Runne, D. Mechanochemical reductive dehalogenation of hazardous polyhalogenated contaminants. J. Mater. Sci. 2004, 39, 5111-5116. [CrossRef]

16. Nah, W.; Hwang, K.-Y.; Shul, Y.-G. Effect of metal and glycol on mechanochemical dechlorination of polychlorinated biphenyls (PCBs). Chemosphere 2008, 73, 138-141. [CrossRef] [PubMed]

17. Bruere, P. Alterations by light, heat and agitation of the heavy petroleum oils refined for therapeutic use. Bull. Travaux Societe de Pharmacie Bordeaux 1928, 9, 142-144.

18. Minami, I. Ionic liquids in tribology. Molecules 2009, 14, 2286-2305. [CrossRef] [PubMed]

19. Chao, K.K.; Saba, C.S. Tribo-Evaluation of High Temperature Candidate Fluids in a Sliding “TBOD” Bench Tester. Tribol. Trans. 1995, 38, 63-68. [CrossRef]

20. Philippon, D.; De Barros-Bouchet, M.-I.; Le Mognea, T.; Lerasle, O.; Bouffet, A.; Martin, J.-M. Role of nascent metallic surfaces on the tribochemistry of phosphite lubricant additives. Tribol. Int. 2011, 44, 684-691. [CrossRef]

21. Warsaw, C.K.; Furey, M.J.; Ritter, A.L.; Molina, G.J. Triboemission as a basic part of the boundary friction regime: A review. Lubr. Sci. 2002, 14, 223-254. [CrossRef]

22. Wang, Q.J.; Zhu, D. "Hertz Theory: Contact of Spherical Surfaces" in Encyclopedia of Tribology; Wang, Q.J., Chung, Y.W., Eds.; Springer: New York, NY, USA, 2013; Volume 5.

23. Schettino, V.; Bini, R. Molecules under extreme conditions: Chemical reactions at high pressure. Phys. Chem. Chem. Phys. 2003, 5, 1951-1965. [CrossRef]

24. Cann, P.M.; Spikes, H.A. In Lubro Studies of Lubricants in EHD Contact Using FTIR Absorption Spectroscopy. Tribol. Trans. 1991, 34, 248-256. [CrossRef]

25. Kauzmann, W.; Eyring, H. The Viscous Flow of Large Molecules. J. Am. Chem. Soc. 1940, 62, 3113-3125. [CrossRef]

26. Carnes, C. The ten greatest events in tribology history. Tribol. Lubr. Technol. 2005, 61, 38-47.

27. Papke, B.L. "Mineral Oil Base Fluids" in Encyclopedia of Tribology; Wang, Q.J., Chung, Y.W., Eds.; Springer: New York, NY, USA, 2013; Volume 4.

28. Michael, J. Covitch Viscosity Index Additives in Encyclopedia of Tribology; Wang, Q.J., Chung, Y.W., Eds.; Springer: New York, NY, USA, 2013; Volume 2.

29. Gresham, R.M. Viscosity index's new importance. Tribol. Lubr. Technol. 2017, 73, 18-19.

30. Nyberg, E.; Respatiningsih, C.Y.; Minami, I. Molecular design of advanced lubricant base fluids: Hydrocarbon-mimicking ionic liquids. RSC Adv. 2017, 7, 6364-6373. [CrossRef]

31. Hardy, W.B.; Doubleday, I. Boundary Lubrication. The Paraffin Series. Proc. R. Soc. Lond. Ser. A 1922, 100, 550-574. [CrossRef]

32. Papay, A.G. Antiwear and Extreme-Pressure Additives in Lubricants. Lubr. Sci. 1998, 10, 209-224. [CrossRef]

33. Rizvi, S.Q.A. Additives for Automotive Fuels and Lubricants. Lubr. Eng. 1999, 55, 33-39.

34. Taylor, R.I. Tribology and energy efficiency: From molecules to lubricated contacts to complete machines. Faraday Discuss. 2012, 156, 361-382. [CrossRef] [PubMed]

35. Bowden, F.P.; Gregory, J.N.; Tabor, D. Lubrication of metal Surfaces by Fatty Acids. Nature 1945, 156, 97-101. [CrossRef]

36. Spikes, H.A. Friction Modifier Additives. Tribol. Lett. 2015, 60, 1-26. [CrossRef] 
37. Rowe, G.W. The chemistry of tribology, friction, lubrication and wear. R. Inst. Chem. Rev. 1968, 1, 135-204. [CrossRef]

38. Loehlé, S.; Matta, C.; Minfray, C.; le Mogne, T.; Iovine, R.; Obara, Y.; Miyamoto, A.; Martin, J.M. Mixed lubrication of steel by $\mathrm{C} 18$ fatty acids revisited. Part I: Toward the formation of carboxylate. Tribol. Int. 2015, 82, 218-227. [CrossRef]

39. Jahanmir, S. Chain Length Effects in Boundary Lubrication. Wear 1985, 102, 331-349. [CrossRef]

40. Spikes, H.A. Film-forming additives-Direct and indirect ways to reduce friction. Lubr. Sci. 2002, 14, $147-167$. [CrossRef]

41. Campen, S.; Green, J.H.; Lamb, G.D.; Spikes, H.A. In Situ Study of Model Organic Friction Modifiers Using Liquid Cell AFM; Saturated and Mono-unsaturated Carboxylic Acids. Tribol. Lett. 2015, 57, 18. [CrossRef]

42. Minami, I.; Kubo, T.; Nanao, H.; Mori, S.; Okuda, S.; Sagawa, T. Investigation of Tribo-Chemistry by Means of Stable Isotopic Tracers, Part 2: Lubrication Mechanism of Friction Modifiers on Diamond-Like Carbon. Tribol. Trans. 2007, 50, 477-487. [CrossRef]

43. Gellman, A.J.; Spencer, N.D. Surface chemistry in tribology, Proceedings of the Institution of Mechanical Engineers, Part J. J. Eng. Tribol. 2002, 216, 443-461.

44. Haider, J. "MoSx Coatings by Closed-Field Magnetron Sputtering" in Encyclopedia of Tribology; Wang, Q.J., Chung, Y.W., Eds.; Springer: New York, NY, USA, 2013; Volume 2.

45. Mitchell, P.C.H. Oil-Soluble Mo-S Compounds as Lubricant Additives. Wear 1984, 100, 281-300. [CrossRef]

46. Graham, J.; Spikes, H.; Korcek, S. The Friction Reducing Properties of Molybdenum Dialkyldithiocarbamate Additives: Part I-Factors Influencing Friction Reduction. Tribol. Trans. 2001, 44, 626-636. [CrossRef]

47. Graham, J.; Spikes, H.; Korcek, S. The Friction Reducing Properties of Molybdenum Dialkyldithiocarbamate Additives: Part II—Durability of Friction Reducing Capability. Tribol. Trans. 2001, 44, 637-647. [CrossRef]

48. Sunqing, Q.; Junxiu, D.; Guoxu, C. A Review of Ultrafine Particle as Antiwear Additives and Friction Modifiers in Lubricating Oils. Lubr. Sci. 1999, 11, 217-226. [CrossRef]

49. Tevet, O.; Von-Huth, P.; Popovitz-Biro, R.; Rosentsveig, R.; Wagner, H.D.; Tenne, R. Friction mechanism of individual multilayered nanoparticles. Proc. Natl. Acad. Sci. USA 2011, 108, 19901-19906. [CrossRef] [PubMed]

50. Joly-Pottuz, L. "Nanolubricants" in Encyclopedia of Tribology; Wang, Q.J., Chung, Y.W., Eds.; Springer: New York, NY, USA, 2013; Volume 4.

51. Wang, X.-B.; Liu, W.-M. "Nanoparticle-Based Lubricant Additives" in Encyclopedia of Tribology; Wang, Q.J., Chung, Y.W., Eds.; Springer: New York, NY, USA, 2013; Volume 4.

52. Daia, W.; Kheireddinb, B.; Gaob, H.; Lianga, H. Roles of nanoparticles in oil lubrication. Tribol. Int. 2016, 102, 88-98. [CrossRef]

53. Guan, B.; Pochopien, B.A.; Wright, D.S. The chemistry, mechanism and function of tricresyl phosphate (TCP) as an anti-wear lubricant additive. Lubr. Sci. 2016, 28, 257-265. [CrossRef]

54. Spikes, H.A. The history and mechanisms of ZDDP. Tribol. Lett. 2004, 17, 469-489. [CrossRef]

55. Nicholls, M.A.; Do, T.; Norton, P.R.; Kasrai, M.; Bancroft, G.M. Review of the lubrication of metallic surfaces by zinc dialkyl-dithiophosphates. Tribol. Int. 2005, 38, 15-39. [CrossRef]

56. Cen, H.; Morina, A.; Neville, A.; Pasaribu, R.; Nedelcu, I. Effect of water on ZDDP anti-wear performance and related tribochemistry in lubricated steel/steel pure sliding contacts. Tribol. Int. 2012, 56, 47-57. [CrossRef]

57. Parsaeian, P.; Ghanbarzadeh, A.; Eijk, M.C.P.V.; Nedelcu, I.; Neville, A.; Morina, A. A new insight into the interfacial mechanisms of the tribofilm formed by zinc dialkyl dithiophosphate. Appl. Surf. Sci. 2017, 403, 472-486. [CrossRef]

58. Parsaeian, P.; Ghanbarzadeh, A.; Wilson, M.; Eijk, M.C.P.V.; Nedelcu, I.; Dowson, D.; Neville, A.; Morina, A. An experimental and analytical study of the effect of water and its tribochemistry on the tribocorrosive wear of boundary lubricated systems with ZDDP-containing oil. Wear 2016, 358-359, 23-31. [CrossRef]

59. Parsaeian, P.; Eijk, M.C.P.V.; Nedelcu, I.; Neville, A.; Morina, A. Study of the interfacial mechanism of ZDDP tribofilm in humid environment and its effect on tribochemical wear; Part I: Experimental. Tribol. Int. 2017, 107, 135-143. [CrossRef]

60. Onodera, T.; Martin, J.-M.; Minfray, C.; Dassenoy, F.; Miyamoto, A. Antiwear Chemistry of ZDDP: Coupling Classical MD and Tight-Binding Quantum Chemical MD Methods (TB-QCMD). Tribol. Lett. 2013, 50, 31-39. [CrossRef] 
61. Grrossiord, C.; Martin, J.-M.; Varlot, K.; Vacher, B.; Mogne, T.L.; Yamada, Y. Tribological interactions between Zndtp, Modtc and calcium borate. Tribol. Lett. 2000, 8, 203-212. [CrossRef]

62. De Barros, M.I.; Bouchet, J.; Raoult, I.; Le Mogne, T.; Martin, J.M.; Kasrai, M.; Yamada, Y. Friction reduction by metal sulfides in boundary lubrication studied by XPS and XANES analyses. Wear 2003, 254, 863-870. [CrossRef]

63. Khaemba, D.N.; Neville, A.; Morina, A. New insights on the decomposition mechanism of Molybdenum DialkyldiThioCarbamate (MoDTC): A Raman spectroscopic study. RSC Adv. 2016, 6, 38637-38646. [CrossRef]

64. Khaemba, D.N.; Jarnias, F.; Thiebaut, B.; Neville, A.; Morina, A. The role of surface roughness and slide-roll ratio on the decomposition of MoDTC in tribological contacts. J. Phys. D Appl. Phys. 2017, 50, 085302. [CrossRef]

65. Allum, K.G.; Forbes, E.S. The Load-carrying Properties of Organic Sulphur Compounds. Part II. The Influence of Chemical Structure on the Anti-wear Properies of Organic Disulphides. J. Inst. Petrol. 1967, 53, 173-185.

66. Covitch, M.J.; Weiss, J.; Kreutzer, I.M. Low-temperature rheology of engine lubricants subjected to mechanical shear: Viscosity modifier effects. Lubr. Sci. 1999, 11, 337-364. [CrossRef]

67. Snyder, C.E., Jr.; Gschwender, L.J.; Paciorek, K.; Kratzer, R.; Nakahra, J. Development of a Shear Stable Viscosity-Index Improver for Use in Hydrogenated Polyalphaolefin-Based Fluids. Lubr. Eng. 1986, 42, 547-557.

68. Souchik, J. "Pour Point Depressants" in Lubricant Additives, 2nd ed.; Rudnick, L.R., Ed.; Marcel Dekker, Inc.: New York, NY, USA, 2009; pp. 339-353.

69. Ingold, K.U. Inhibition of the Autoxidation of Organic Substrates in the Liquid Phase. Chem. Rev. 1961, 6, 563-589. [CrossRef]

70. Jensen, R.K.; Korcek, S.; Mahoney, L.R.; Zinbo, M. Liquid-Phase Autoxidation of Organic Compounds at Elevated Temperaturess. 1. The Stirred Flow Reactor Technique and Analysis of Primary Products from n-Hexadecane Autoxidation at $120-180^{\circ}$ C. J. Am. Chem. Soc. 1979, 101, 7574-7584. [CrossRef]

71. Jensen, R.K.; Korcek, S.; Mahoney, L.R.; Zinbo, M. Liquid-Phase Autoxidation of Organic Compounds at ElevatedTemperaturess. 2. Kinetics and Mechanisms of the Formation of Cleavage Products n-Hexadecane. J. Am. Chem. Soc. 1981, 103, 1742-1749. [CrossRef]

72. Jensen, R.K.; Korcek, S.; Zinbo, M.; Jensen, M.D. Initiation in Hydrocarbon Autoxidation at Elevated Temperatures. Int. J. Chem. Kinet. 1990, 22, 1095-1107. [CrossRef]

73. Maleville, X.; Faure, D.; Legros, A.; Hipeaux, J.C. Oxidation of mineral base oils of petroleum origin: The relationship between chemical composition, thickening, and composition of degradation products. Lubr. Sci. 1996, 9, 3-60. [CrossRef]

74. Horswill, E.C.; Ingold, K.U. The Oxidation of Phenols I. The Oxidation of 2,6-Di-t-butyl-4-methylphenol, 2,6-Di-t-butylphenol, and2,6-Dimethylphenol with Peroxy Radicals. Can. J. Chem. 1966, 44, 263-268. [CrossRef]

75. Horswill, E.C.; Ingold, K.U. The Oxidation of Phenols II. The Oxidation of 2,4-Di-t-butylphenol with Peroxy Radicals. Can. J. Chem. 1966, 44, 269-277. [CrossRef]

76. Dong, J.; Migdal, C.A. "Antioxidants" in Lubricant Additives, 2nd ed.; Rudnick, L.R., Ed.; Marcel Dekker, Inc.: New York, NY, USA, 2009; pp. 3-50.

77. Zeman, A.; Romer, R.; Roenne, V. Fate of Amine Antioxidants During Thermal Oxidative Aging of Neopentylpolyol Ester Oils: Part 1. J. Synth. Lubr. 1987, 3, 309-326. [CrossRef]

78. Zeman, A.; Roenne, V.; Trebert, Y. Fate of Amine Antioxidants During Thermal Oxidation Ageing of Neopentylpolyl Ester Oils. J. Synth. Lubr. 1987, 4, 179-201. [CrossRef]

79. Holdsworth, J.D.; Scott, G.; Williams, D. Mechanism of Antioxidant Action: Sulpur-containing Antioxidants. J. Chem. Soc. 1964, 1964, 4692-4699. [CrossRef]

80. Burn, A.J. The Mechanism of the Antioxidant Action of Zinc Dialkyl Dithiophosphates. Tetrahedron 1966, 22, 2153-2161. [CrossRef]

81. Gatto, V.J.; Moehle, W.E.; Cobb, T.W.; Schneller, E.R. The relationship between oxidation stability and antioxidant depletion in turbine oils formulated with Groups II, III and IV base stocks. J. Synth. Lubr. 2007, 24, 111-124. [CrossRef]

82. O'Connor, S.P.; Crawford, J.; Cane, C. Overbased Lubricant Detergents-A Comparative Study. Lubr. Sci. 1994, 6, 297-325. [CrossRef]

83. Olomolehin, Y.; Kapadia, R.; Spikes, H. Antagonistic Interaction of Antiwear Additives and Carbon Black. Tribol. Lett. 2010, 37, 49-58. [CrossRef] 
84. Bartha, L.; deak, G.; Baladincz, J.; Auer, J.; Kocsis, Z. Polyfunctional PIB Succinimide Type Engine Oil Additives. Lubr. Sci. 2001, 13, 313-328. [CrossRef]

85. Costello, M.T. "Corrosion Inhibitors and Rust Preventatives" in Lubricant Additives, 2nd ed.; Rudnick, L.R., Ed.; Marcel Dekker, Inc.: New York, NY, USA, 2009; pp. 421-444.

86. Duncanson, M. Effects of Physical and Chemical Properties on Foam in Lubricating Oil. Lubr. Eng. 2003, 59, 9-13.

87. Centers, P.W. Behavior of Silicone Antifoam Additives in Synthetic Ester Lubricants. Tribol. Trans. 1993, 36, 381-386. [CrossRef]

88. Rowe, C.N.; Dickert, J.J. The Relation of Antiwear Function to Thermal Stability and Structure for Metal O,O-Dialkylphosphorodithioates. ASLE Trans. 1967, 10, 85-90. [CrossRef]

89. McDonald, R.A. "Zinc Dithiophosphates" in Lubricant Additives, 2nd ed.; Rudnick, L.R., Ed.; Marcel Dekker, Inc.: New York, NY, USA, 2009; pp. 51-62.

90. Hong, H.; Riga, A.T.; Cahoon, J.M.; Vinci, J.N. Evaluation of Overbased Sulfonates as Extreme-Pressure Additives in Metalworking Fluids. Lubr. Eng. 1993, 49, 19-24.

91. Keromest, C.; Durand, J.-P.; Born, M.; Gateau, P.; Tessier, M.; Marechal, E. Phosphosulphurised Antiwear and Extreme Pressure VI Improver Polymer Additives: Synthesis, Properties, and Lubricant Applications. Lubr. Sci. 1988, 10, 179-197. [CrossRef]

92. Ota, J.; Hait, S.K.; Sastry, M.I.S.; Ramakumar, S.S.V. Graphene dispersion in hydrocarbon medium and its application in lubricant technology. RSC Adv. 2015, 5, 53326-53332. [CrossRef]

93. Minami, I.; Hong, H.S.; Mathur, N.C. Lubrication Performance of Model Organic Compounds in High Oleic Sunflower oil. J. Synth. Lubr. 1999, 16, 3-12. [CrossRef]

94. Palacios, J.M. The Performance of some Antiwear Additives and Interference with Other Additives. Lubr. Sci. 1992, 4, 201-209. [CrossRef]

95. Guerret-Piecourt, C.; Grossiord, C.; Mogne, T.L.; Martin, J.M.; Palermo, T. Role of Complexation in the Interaction between Antiwear and Dispersant Additives in Lubricants. Lubr. Sci. 2001, 13, 201-218. [CrossRef]

96. Kuhlman, R.E. "Environmentally Friendly Lubrication Issues" in Encyclopedia of Tribology; Wang, Q.J., Chung, Y.W., Eds.; Springer: New York, NY, USA, 2013; Volume 2, pp. 985-991.

97. Minami, I.; Kikuta, S.; Okabe, H. Anti-wear and friction reducing additives composed of ortho-phenylene phosphate-amine salts for polyether type base stocks. Tribol. Int. 1998, 31, 305-312. [CrossRef]

98. Van der Waal, G. The Relationship between the Chemical Structure of Ester Base Fluids and their Influence on Elastomer Seals, and Wear Characteristics. J. Synth. Lubr. 1985, 1, 280-301. [CrossRef]

99. Minami, I.; Mori, S. Concept of molecular design towards additive technology for advanced lubricants. Lubr. Sci. 2007, 19, 127-149. [CrossRef]

100. Jessop, P.G.; Ahmadpour, F.; Buczynski, M.A.; Burns, T.J.; Green, N.B., II; Korwin, R.; Long, D.; Massad, S.K.; Manley, J.B.; Omidbakhsh, N.; et al. Opportunities for greener alternatives in chemical formulations. Green Chem. 2015, 17, 2664-2678. [CrossRef]

(C) 2017 by the author. Licensee MDPI, Basel, Switzerland. This article is an open access article distributed under the terms and conditions of the Creative Commons Attribution (CC BY) license (http://creativecommons.org/licenses/by/4.0/). 